\title{
Planck 2013 results. XXV. Searches for cosmic strings and other topological defects
}

\begin{abstract}
Planck Collaboration: P. A. R. Ade ${ }^{83}$, N. Aghanim ${ }^{55}$, C. Armitage-Caplan ${ }^{88}$, M. Arnaud ${ }^{69}$, M. Ashdown ${ }^{66,6}$, F. Atrio-Barandela $^{18}$, J. Aumont $^{55}$ C. Baccigalupi ${ }^{82}$, A. J. Banday ${ }^{91,9}$, R. B. Barreiro ${ }^{63}$, J. G. Bartlett ${ }^{1,64}$, N. Bartolo ${ }^{31}$, E. Battaner ${ }^{92}$, R. Battye ${ }^{65}$, K. Benabed ${ }^{56,90}$, A. Benoît ${ }^{53}$, A. Benoit-Lévy ${ }^{25,56,90}$, J.-P. Bernard ${ }^{9}$, M. Bersanelli 34,46, P. Bielewicz ${ }^{919,82}$, J. Bobin ${ }^{69}$, J. J. Bock ${ }^{64,10}$, A. Bonaldi ${ }^{65}$, L. Bonavera ${ }^{63}$, J. R. Bond ${ }^{8}$, J. Borrill ${ }^{13,85}$, F. R. Bouchet ${ }^{56,90}$, M. Bridges ${ }^{66,6,59}$, M. Bucher ${ }^{1}$, C. Burigana ${ }^{45,32}$, R. C. Butler ${ }^{45}$, J.-F. Cardoso ${ }^{70,1,56}$, A. Catalano ${ }^{71,68}$, A. Challinor ${ }^{59,66,11}$, A. Chamballu ${ }^{69,15,55}$, L.-Y Chiang ${ }^{58}$, H. C. Chiang ${ }^{27,7}$, P. R. Christensen ${ }^{78,37}$, S. Church ${ }^{87}$, D. L. Clements ${ }^{51}$, S. Colombi ${ }^{56,90}$, L. P. L. Colombo ${ }^{24,64}$, F. Couchot ${ }^{67}$, A. Coulais ${ }^{68}$, B. P. Crill ${ }^{64,79}$, A. Curto ${ }^{6,63}$, F. Cuttaia ${ }^{45}$, L. Danese ${ }^{82}$, R. D. Davies ${ }^{65}$, R. J. Davis ${ }^{65}$, P. de Bernardis ${ }^{33}$, A. de Rosa ${ }^{45}$, G. de Zotti ${ }^{42,82}$, J. Delabrouille ${ }^{1}$, J.-M. Delouis ${ }^{56,90}$, F.-X. Désert ${ }^{49}$, J. M. Diego ${ }^{63}$, H. Dole ${ }^{55,54}$, S. Donzelli ${ }^{46}$, O. Doré ${ }^{64,10}$, M. Douspis ${ }^{55}$, A. Ducout ${ }^{56}$, J. Dunkley ${ }^{88}$, X. Dupac ${ }^{39}$, G. Efstathiou ${ }^{59}$, T. A. Enßlin ${ }^{74}$, H. K. Eriksen ${ }^{61}$, J. Fergusson ${ }^{11}$, F. Finellii ${ }^{45,47}$,

O. Forni ${ }^{91,9}$, M. Frailis ${ }^{44}$, E. Franceschi ${ }^{45}$, S. Galeotta ${ }^{44}$, K. Ganga ${ }^{1}$, M. Giard ${ }^{91,9}$, G. Giardino ${ }^{40}$, Y. Giraud-Héraud ${ }^{1}$, J. González-Nuevo ${ }^{63,82}$, K. M. Górski ${ }^{64,94}$, S. Gratton ${ }^{66,59}$, A. Gregorio ${ }^{35,44}$, A. Gruppuso ${ }^{45}$, F. K. Hansen ${ }^{61}$, D. Hanson ${ }^{76,64,8}$, D. Harrison ${ }^{59,66}$, S. Henrot-Versillé67, $^{67}$ C. Hernández-Monteagudo ${ }^{12,74}$, D. Herranz ${ }^{63}$, S. R. Hildebrandt ${ }^{10}$, E. Hivon ${ }^{56,90}$, M. Hobson ${ }^{6}$, W. A. Holmes ${ }^{64}$, A. Hornstrup ${ }^{16}$, W. Hovest ${ }^{74}$, K. M. Huffenberger ${ }^{93}$, T. R. Jaffe ${ }^{91,9}$, A. H. Jaffe ${ }^{51}$, W. C. Jones ${ }^{27}$, M. Juvela ${ }^{26}$, E. Keihänen ${ }^{26}$, R. Keskitalo ${ }^{22,13}$, T. S. Kisner ${ }^{73}$, J. Knoche ${ }^{74}$, L. $\operatorname{Knox}^{28}$, M. Kunz ${ }^{17,55,3}$, H. Kurki-Suonio ${ }^{26,41}$, G. Lagache ${ }^{55}$, A. Lähteenmäki ${ }^{2,41}$, J.-M. Lamarre ${ }^{68}$, A. Lasenby ${ }^{6,66}$, R. J. Laureijs ${ }^{40}$, C. R. Lawrence ${ }^{64}$, J. P. Leahy ${ }^{65}$, R. Leonardi ${ }^{39}$, J. Lesgourgues ${ }^{89,81}$, M. Liguori ${ }^{31}$, P. B. Lilje ${ }^{61}$, M. Linden-Vørnle ${ }^{16}$, M. López-Caniego ${ }^{63}$, P. M. Lubin ${ }^{29}$, J. F. Macías-Pérez ${ }^{71}$, B. Maffei ${ }^{65}$, D. Maino ${ }^{34,46}$, N. Mandolesi ${ }^{45,5,32}$, M. Maris ${ }^{44}$, D. J. Marshall ${ }^{69}$, P. G. Martin ${ }^{8}$,

E. Martínez-González ${ }^{63}$, S. Masi ${ }^{33}$, S. Matarrese ${ }^{31}$, F. Matthai ${ }^{74}$, P. Mazzotta $^{36}$, J. D. McEwen ${ }^{25}$, A. Melchiorri ${ }^{33,48}$, L. Mendes ${ }^{39}$, A. Mennella ${ }^{34,46}$, M. Migliaccio ${ }^{59,66}$, S. Mitra ${ }^{50,64}$, M.-A. Miville-Deschênes ${ }^{55,8}$, A. Moneti ${ }^{56}$, L. Montier ${ }^{91,9}$, G. Morgante ${ }^{45}$, D. Mortlock ${ }^{51}$, A. Moss $^{84, \star}$, D. Munshi ${ }^{83}$, P. Naselsky ${ }^{78,37}$, P. Natoli ${ }^{32,4,45}$, C. B. Netterfield ${ }^{20}$, H. U. Nørgaard-Nielsen ${ }^{16}$, F. Noviello ${ }^{65}$, D. Novikov ${ }^{51}$, I. Novikov ${ }^{78}$, S. Osborne ${ }^{87}$, C. A. Oxborrow ${ }^{16}$, F. Paci ${ }^{82}$, L. Pagano ${ }^{33,48}$, F. Pajot ${ }^{55}$, D. Paoletti ${ }^{45,47}$, F. Pasian ${ }^{44}$, G. Patanchon ${ }^{1}$, H. V. Peiris ${ }^{25}$, O. Perdereau ${ }^{67}$, L. Perotto ${ }^{71}$, F. Perrotta ${ }^{82}$, F. Piacentini ${ }^{33}$, M. Piat ${ }^{1}$, E. Pierpaoli ${ }^{24}$, D. Pietrobon ${ }^{64}$, S. Plaszczynski ${ }^{67}$, E. Pointecouteau ${ }^{91,9}$, G. Polenta ${ }^{4,43}$, N. Ponthieu ${ }^{55,49}$, L. Popa ${ }^{57}$, T. Poutanen ${ }^{41,26,2}$, G. W. Pratt ${ }^{69}$, G. Prézeau ${ }^{10,64}$, S. Prunet ${ }^{56,90}$, J.-L. Puget ${ }^{55}$, J. P. Rachen ${ }^{21,74}$, C. Räth ${ }^{75}$, R. Rebolo ${ }^{62,14,38}$, M. Remazeilles ${ }^{55,1}$, C. Renault ${ }^{71}$, S. Ricciardi ${ }^{45}$, T. Riller ${ }^{74}$, C. Ringeval ${ }^{60,56,90}$, I. Ristorcelli ${ }^{11,9}$, G. Rocha $^{64,10}$, C. Rosset ${ }^{1}$, G. Roudier ${ }^{1,68,64}$, M. Rowan-Robinson ${ }^{51}$, B. Rusholme ${ }^{52}$, M. Sandri ${ }^{45}$, D. Santos ${ }^{71}$, G. Savini ${ }^{80}$, D. Scott ${ }^{23}$, M. D. Seiffert ${ }^{64,10}$, E. P. S. Shellard ${ }^{11}$, L. D. Spencer ${ }^{83}$, J.-L. Starck ${ }^{69}$, V. Stolyarov ${ }^{6,66,86}$, R. Stompor ${ }^{1}$, R. Sudiwala ${ }^{83}$, F. Sureau ${ }^{69}$, D. Sutton ${ }^{59,66}$,
\end{abstract} A.-S. Suur-Uski ${ }^{26,41}$, J.-F. Sygnet ${ }^{56}$, J. A. Tauber ${ }^{40}$, D. Tavagnacco ${ }^{44,35}$, L. Terenzi ${ }^{45}$, L. Toffolatti ${ }^{19,63}$, M. Tomasi ${ }^{46}$, M. Tristram $^{67}$, M. Tucci $^{17,67}$, J. Tuovinen ${ }^{77}$, L. Valenziano ${ }^{45}$, J. Valiviita ${ }^{41,26,61}$, B. Van Tent ${ }^{72}$, J. Varis ${ }^{77}$, P. Vielva ${ }^{63}$, F. Villa ${ }^{45}$, N. Vittorio ${ }^{36}$, L. A. Wade ${ }^{64}$, B. D. Wandelt ${ }^{56,90,30}$, D. Yvon ${ }^{15}$, A. Zacchei ${ }^{44}$, and A. Zonca ${ }^{29}$

(Affiliations can be found after the references)

Received 1 April 2013 / Accepted 23 January 2014

\begin{abstract}
Planck data have been used to provide stringent new constraints on cosmic strings and other defects. We describe forecasts of the CMB power spectrum induced by cosmic strings, calculating these from network models and simulations using line-of-sight Boltzmann solvers. We have studied Nambu-Goto cosmic strings, as well as field theory strings for which radiative effects are important, thus spanning the range of theoretical uncertainty in the underlying strings models. We have added the angular power spectrum from strings to that for a simple adiabatic model, with the extra fraction defined as $f_{10}$ at multipole $\ell=10$. This parameter has been added to the standard six parameter fit using COSMOMC with flat priors. For the Nambu-Goto string model, we have obtained a constraint on the string tension of $G \mu / c^{2}<1.5 \times 10^{-7}$ and $f_{10}<0.015$ at $95 \%$ confidence that can be improved to $G \mu / c^{2}<1.3 \times 10^{-7}$ and $f_{10}<0.010$ on inclusion of high- $\ell$ CMB data. For the Abelian-Higgs field theory model we find, $G \mu_{\mathrm{AH}} / c^{2}<3.2 \times 10^{-7}$ and $f_{10}<0.028$. The marginalised likelihoods for $f_{10}$ and in the $f_{10}-\Omega_{\mathrm{b}} h^{2}$ plane are also presented. We have additionally obtained comparable constraints on $f_{10}$ for models with semilocal strings and global textures. In terms of the effective defect energy scale these are somewhat weaker at $G \mu / c^{2}<1.1 \times 10^{-6}$. We have made complementarity searches for the specific non-Gaussian signatures of cosmic strings, calibrating with all-sky Planck resolution CMB maps generated from networks of post-recombination strings. We have validated our non-Gaussian searches using these simulated maps in a Planck-realistic context, estimating sensitivities of up to $\Delta G \mu / c^{2} \approx 4 \times 10^{-7}$. We have obtained upper limits on the string tension at $95 \%$ confidence of $G \mu / c^{2}<9.0 \times 10^{-7}$ with modal bispectrum estimation and $G \mu / c^{2}<7.8 \times 10^{-7}$ for real space searches with Minkowski functionals. These are conservative upper bounds because only post-recombination string contributions have been included in the non-Gaussian analysis.
\end{abstract}

Key words. cosmic background radiation - cosmological parameters - early Universe - large-scale structure of Universe - cosmology: theory cosmology: observations

^ Corresponding author: A. Moss

e-mail: adam.moss@nottingham.ac.uk 


\section{Introduction}

This paper, one of a set associated with the 2013 release of data from the Planck ${ }^{1}$ mission (Planck Collaboration I 2014), describes the constraints on cosmic strings, semi-local strings and global textures. Such cosmic defects are a generic outcome of symmetry-breaking phase transitions in the early Universe (Kibble 1976) and further motivation came from a potential role in large-scale structure formation (Zeldovich 1980; Vilenkin 1981a). Cosmic strings appear in a variety of supersymmetric and other grand unified theories, forming at the end of inflation (see, for example, Jeannerot et al. 2003). However, further interest in cosmic (super-)strings has been motivated by their emergence in higher-dimensional theories for the origin of our Universe, such as brane inflation. These superstring variants come in a number of $D$ - and $F$-string forms, creating hybrid networks with more complex dynamics (see, e.g., Polchinski 2005). Cosmic strings can have an enormous energy per unit length $\mu$ that can give rise to a number of observable effects, including gravitational lensing and a background of gravitational waves. Here, we shall concentrate on the impact of strings on the cosmic microwave background (CMB), which includes the generation of line-like discontinuities in temperature. Comparable effects can also be caused by other types of cosmic defects, notably semi-local strings and global textures. As well as influencing the CMB power spectrum, each type of topological defect should have a counterpart non-Gaussian signature giving us the ability distinguish between different defects, alternative scenarios, or systematic effects. The discovery of any of these objects would profoundly influence our understanding of fundamental physics, identifying GUT-scale symmetry breaking patterns, perhaps even providing direct evidence for extra dimensions. Conversely, the absence of these objects will tightly constrain symmetry breaking schemes, again providing guidance for high energy theory. For a general introduction to cosmic strings and other defects, refer to Vilenkin \& Shellard (2000); Hindmarsh \& Kibble (1995); Copeland \& Kibble (2010).

High resolution numerical simulations of cosmic strings using the Nambu-Goto action indicate that cosmological networks tend towards a scale-invariant solution with typically tens of long strings stretching across each horizon volume. These strings continuously source gravitational perturbations on sub-horizon scales, the magnitude of that are determined by the dimensionless parameter:

$$
\frac{G \mu}{c^{2}}=\left(\frac{\eta}{m_{\mathrm{Pl}}}\right)^{2}
$$

where $\eta$ is the energy scale of the string-forming phase transition and $m_{\mathrm{Pl}} \equiv \sqrt{\hbar c / G}$ is the Planck mass. String effects on the $\mathrm{CMB}$ power spectrum have been estimated using a phenomenological string model and, with WMAP and SDSS data, these estimates yield a $2 \sigma$ upper bound of $G \mu / c^{2}<2.6 \times 10^{-7}$ (Battye $\&$ Moss 2010). A consequence is that strings can be responsible for no more than $4.4 \%$ of the CMB anisotropy signal at multipole $\ell=10$.

As we shall discuss, the evolution of Nambu-Goto string networks is computationally challenging and quantitative

\footnotetext{
1 Planck (http://www.esa.int/Planck) is a project of the European Space Agency (ESA) with instruments provided by two scientific consortia funded by ESA member states (in particular the lead countries France and Italy), with contributions from NASA (USA) and telescope reflectors provided by a collaboration between ESA and a scientific consortium led and funded by Denmark.
}

uncertainties remain, notably in characterising the string smallscale structure and loop production. An alternative approach has been to use field theory simulations of cosmological vortexstrings. These yield a significantly lower number of strings per horizon volume (less than half), reflecting the importance of radiative effects on the microphysical scales being probed numerically. The degree of convergence with Nambu-Goto string simulations is difficult to determine computationally at present, but there are also global strings for which radiative effects of comparable magnitude are expected to remain important on cosmological scales. It is prudent in this paper, therefore, to constrain both varieties of strings, labelling the field theory constraints as $\mathrm{AH}$ from the Abelian-Higgs (local $U(1))$ model used to describe them. Given these quantitative differences, such as the lower density, field theory strings produce a weaker constraint $G \mu_{\mathrm{AH}} / c^{2}<5.7 \times 10^{-7}$ using WMAP data alone (Bevis et al. 2008) and (Urrestilla et al. 2011). The shape of the stringinduced power spectrum also has a different shape, which allows up to a $9.5 \%$ contribution at $\ell=10$. These WMAP constraints can be improved by adding small-scale CMB anisotropy in a joint analysis. The Nambu-Goto strings limit improves to become $G \mu / c^{2}<1.7 \times 10^{-7}$ (using SPT data, Dvorkin et al. 2011) and field theory strings yield $G \mu_{\mathrm{AH}} / c^{2}<4.2 \times 10^{-7}$ (Urrestilla et al. 2011). Power-spectrum based constraints on global textures were studied in Bevis et al. (2004) and (Urrestilla et al. 2008), with the latter paper giving a $95 \%$ limit of $G \mu / c^{2}<4.5 \times 10^{-6}$. Urrestilla et al. (2008) also provide constraints on semi-local strings, $G \mu / c^{2}<5.3 \times 10^{-6}$.

Constraints on cosmic strings from non-Gaussianity require high resolution realisations of string-induced $\mathrm{CMB}$ maps that are extremely challenging to produce. Low resolution smallangle and full-sky CMB maps calculated with the full recombination physics included, have indicated some evidence for a significant kurtosis from strings (Landriau \& Shellard 2011). More progress has been made in creating high resolution maps from string lensing after recombination (see Ringeval \& Bouchet (2012) and references therein) and identifying, in principle, the bispectrum and trispectrum, which can be predicted for strings analytically (Hindmarsh et al. 2009, 2010; Regan \& Shellard 2010). The first WMAP constraint on cosmic strings using the analytic CMB trispectrum yielded $G \mu / c^{2}<1.1 \times 10^{-6}$ at $95 \%$ confidence (Fergusson et al. 2010b). An alternative approach is to fit pixel-space templates to a map, this method was applied to global textures templates in Cruz et al. (2007) and Feeney et al. (2013, 2012).

The most stringent constraints that are claimed for the string tension arise from predicted backgrounds of gravitational waves that are created by decaying loops (Vilenkin 1981b). However, these constraints are strongly dependent on uncertain string physics, most notably the network loop production scale and the nature of string radiation from cusps, i.e., points on the strings approaching the speed of light $c$. The most optimistic constraint based on the European Pulsar Timing Array is $G \mu / c^{2}<$ $4.0 \times 10^{-9}$ (van Haasteren et al. 2011), but a much more conservative estimate of $G \mu / c^{2}<5.3 \times 10^{-7}$ can be found in Sanidas et al. (2012), together with a string parameter constraint survey and an extensive discussion of these uncertainties. Such gravitational wave limits do not apply to global strings or to strings for which other radiative channels are available.

Alternative topological defects scenarios also have strong motivations and we report limits on textures and global monopoles in this paper as well. Of particular recent interest are hybrid networks of cosmic strings where the correlation length is reduced by having several interacting varieties (e.g., $F$ - and 
$D$-strings) or a small reconnection probability, $p<1$. We expect to investigate these models using the Planck full mission data.

The outline of this paper is as follows. In Sect. 2 we briefly describe the different types of topological defects that we consider, and their impact on the CMB anisotropies. We also discuss how the CMB power spectrum is computed and how we obtain CMB maps with a cosmic string contribution. In Sect. 3 we present the defect constraints from the CMB power spectrum (with numbers given in Table 2), while Sect. 4 discusses searches for topological defects with the help of their non-Gaussian signature. We finally present the overall conclusions in Sect. 5.

\section{Theoretical modelling and forecasting}

\subsection{Cosmic strings and their cosmological consequences}

\subsubsection{String network evolution}

A detailed quantitative understanding of the cosmological evolution of string networks is an essential pre-requisite for making accurate predictions about the cosmological consequences of strings. Fortunately, all string network simulations to date have demonstrated convincingly that the large-scale properties of strings approach a self-similar scale-invariant regime soon after formation. If we treat the string as a one-dimensional object, then it sweeps out a two-dimensional worldsheet in spacetime

$$
x^{\mu}=x^{\mu}\left(\zeta^{a}\right), \quad a=0,1,
$$

where the worldsheet parameters $\zeta^{0}$ and $\zeta^{1}$ are time-like and space-like respectively. The Nambu-Goto action that governs string motion then becomes

$S=-\mu \int \sqrt{-\gamma} \mathrm{d}^{2} \zeta$

where $\gamma_{a b}=g_{\mu \nu} \partial_{a} x^{\mu} \partial_{b} x^{\nu}$ is the two-dimensional worldsheet metric $\left(\gamma=\operatorname{det}\left(\gamma_{a b}\right)\right)$ induced by the spacetime metric $g_{\mu \nu}$. The Nambu-Goto action Eq. (3) can be derived systematically from a field theory action, such as that for the Abelian-Higgs model describing $U(1)$ vortex-strings:

$S=\int \mathrm{d}^{4} x \sqrt{-g}\left[\left(D_{\mu} \phi\right)^{*}\left(D^{\mu} \phi\right)-\frac{1}{4 e^{2}} F_{\mu \nu} F^{\mu \nu}-\frac{\lambda}{4}\left(|\phi|^{2}-\eta^{2}\right)^{2}\right]$,

where $\phi$ is a complex scalar field, $F^{\mu v}$ is the $U(1)$ field strength and $D_{\mu}=\partial_{\mu}+\mathrm{i} A_{\mu}$ is the gauge-covariant derivative with $e$ and $\lambda$ dimensionless coupling constants. The transverse degrees of freedom in $\phi$ can be integrated out provided the string is not strongly curved, that is, the string width $\delta \approx \hbar c / \eta \ll L$ where $L$ is the typical radius of curvature. For a cosmological string network today with $G \mu / c^{2} \sim 10^{-7}$, these two lengthscales are separated by over 40 orders of magnitude, so this should be a valid approximation.

In an expanding universe, the Nambu-Goto action Eq. (3) yields a Hubble-damped wave equation governing the string motion. These equations can be solved numerically, provided "kinks" or velocity discontinuities are treated carefully. However, they can also be averaged analytically to describe the scale-invariant evolution of the whole string network in terms of two quantities, the energy density $\rho$ and the rms velocity $v$. Any string network divides fairly neatly into two distinct populations of long (or "infinite") strings $\rho_{\infty}$ stretching beyond the Hubble radius and the small loops $\rho_{l}$ with length $l \ll H^{-1}$ that the long strings create Kibble (1985). Assuming the long strings form a
Table 1. Summary of numerical simulation results ${ }^{a}$ for the string density parameter $\zeta$ defined in Eq. (5).

\begin{tabular}{crrrrc}
\hline \hline & \multicolumn{5}{c}{$\zeta$} \\
\cline { 2 - 6 } Epoch & MS & RSB & BOS & MSM & BHKU \\
\hline Radiation $\ldots \ldots \ldots$ & 11.5 & 9.5 & 11.0 & 5.0 & 3.8 \\
Matter . . . . . . . & 3.0 & 3.2 & 3.7 & 1.5 & 1.3 \\
\hline
\end{tabular}

Notes. The Nambu-Goto string simulations are respectively labelled as MS (Martins \& Shellard 2006), RSB (Ringeval et al. 2007), and BOS (Blanco-Pillado et al. 2011). This is contrasted with the much lower density results from lattice field theory simulations of vortex-strings labelled as MSM (Moore et al. 2002) and BHKU (Bevis et al. 2007b).

Brownian random walk characterised by a correlation length $L$, we have

$\rho_{\infty}=\frac{\mu}{L^{2}} \equiv \frac{\zeta \mu}{t^{2}}$

and the averaged equations of motion become simply

$\begin{aligned} 2 \frac{\mathrm{d} L}{\mathrm{~d} t} & =2 H L\left(1+v^{2}\right)+\tilde{c} v, \\ \frac{\mathrm{d} v_{\infty}}{\mathrm{d} t} & =\left(1-v^{2}\right)\left[\frac{k(v)}{L}-2 H v\right],\end{aligned}$

where $\tilde{c}$ measures the network loop production rate and $k(v)$ is a curvature parameter with $k \approx 2 \sqrt{2}(1-\sqrt{2} v)$. This is the velocitydependent one-scale (VOS) model and, with a single parameter $\tilde{c}$, it provides a good fit to both Nambu and field theory simulations, notably through the radiation-matter transition (Martins \& Shellard 1996).

A general consensus has emerged from the three main simulation codes describing Nambu-Goto string networks (Martins \& Shellard 2006; Ringeval et al. 2007; Blanco-Pillado et al. 2011). These independent codes essentially solve for left- and rightmoving modes along the string using special techniques to handle contact discontinuities or kinks, including "shock fronting", artificial compression methods and an exact solver for piecewise linear strings, respectively. The consistency between simulations is shown in Table 1 for the string density parameter $\zeta$ defined in Eq. (5). Averaging yields the radiation era density $\zeta=10.7$ and a matter era value $\zeta=3.3$. Note that these asymptotic values and the intervening matter-radiation transition can be welldescribed by the VOS model Eq. (6) with $\tilde{c}=0.23$. The matter era VOS value appears somewhat anomalous from the other two simulations, but this is obtained from larger simulations in a regime where convergence is very slow, so it may more closely reflect the true asymptotic value. These simulations have also advanced the study of string small-scale structure and the loop distribution, about which there had been less consensus (see, e.g., Blanco-Pillado et al. 2011). However, note that CMB anisotropy is far less sensitive to this issue compared to constraints from gravitational waves.

Field theory simulations using lattice gauge techniques have also been employed to study the evolution of string networks in an expanding universe. Comparatively, these three-dimensional simulations are constrained to a lower dynamic range and the simulations require the solution of modified field equations to prevent the string core width shrinking below the lattice resolution. On the other hand, field theory simulations include field radiation and therefore provide a more complete account of the string physics. In Table 1 the lower string densities obtained 
Table 2. 95\% upper limits on the constrained parameter $f_{10}$ and the derived parameter $G \mu / c^{2}$ for the five defect models discussed in the text.

\begin{tabular}{crrrrr}
\hline \hline & \multicolumn{2}{c}{ Planck+WP } & & \multicolumn{2}{c}{ Planck+WP+highL } \\
\cline { 2 - 3 } \cline { 5 - 6 } Defect type & \multicolumn{1}{c}{$f_{10}$} & \multicolumn{1}{c}{$G \mu / c^{2}$} & & $f_{10}$ & \multicolumn{1}{c}{$G \mu / c^{2}$} \\
\hline NAMBU $\ldots \ldots \ldots$ & 0.015 & $1.5 \times 10^{-7}$ & & 0.010 & $1.3 \times 10^{-7}$ \\
AH-mimic $\ldots \ldots \ldots$ & 0.033 & $3.6 \times 10^{-7}$ & & 0.034 & $3.7 \times 10^{-7}$ \\
AH $\ldots \ldots \ldots \ldots$ & 0.028 & $3.2 \times 10^{-7}$ & & 0.024 & $3.0 \times 10^{-7}$ \\
SL $\ldots \ldots \ldots \ldots$ & 0.043 & $11.0 \times 10^{-7}$ & & 0.041 & $10.7 \times 10^{-7}$ \\
TX $\ldots \ldots \ldots \ldots \ldots$ & 0.055 & $10.6 \times 10^{-7}$ & & 0.054 & $10.5 \times 10^{-7}$ \\
\hline
\end{tabular}

Notes. We present limits using Planck and polarisation information from WMAP $($ Planck $+\mathrm{WP})$, and from also including high $\ell \mathrm{CMB}$ information from ACT and SPT (Planck $+\mathrm{WP}+$ highL).

from two sets of Abelian-Higgs simulations are given (Moore et al. 2002; Bevis et al. 2007b). The evolution can be fitted with a VOS model Eq. (6) with $\tilde{c}=0.57$, which is $150 \%$ higher than for Nambu-Goto strings. Field theory simulations have further important applications, particularly for describing delocalised topological defects such as textures, for describing models that do not form stable defects like semilocal strings, and because they include radiative effects naturally. Radiative effects observed in current Abelian-Higgs simulations are comparable to the radiative damping anticipated for cosmological global strings and so the $\mathrm{AH}$ analysis below should offer some insight into this case.

\subsubsection{String gravity and the $\mathrm{CMB}$}

Despite the enormous energy per unit length $\mu$, the spacetime around a straight cosmic string is locally flat. The string has an equation of state $p_{z}=-\rho, p_{x}=p_{y}=0$ (for one lying along the $z$-direction), so there is no source term in the relativistic version of the Poisson equation $\nabla^{2} \Phi=4 \pi G\left(\rho+p_{x}+p_{y}+p_{z}\right)$. The straight string exhibits no analogue of the Newtonian pull of gravity on any surrounding matter. But this does not mean the string has no gravitational impact; on the contrary, a moving string has dramatic effects on nearby matter or propagating CMB photons.

The spacetime metric about a straight static string takes the simple form,

$\mathrm{d} s^{2}=\mathrm{d} t^{2}-\mathrm{d} z^{2}-\mathrm{d} r^{2}-r^{2} \mathrm{~d} \theta^{2}$,

that looks like Minkowski space in cylindrical coordinates, but for the fact that the azimuthal coordinate $\theta$ has a restricted range $0 \leq \theta \leq 2 \pi(1-4 G \mu)$. The spacetime is actually conical with a global deficit angle $\Delta=8 \pi G \mu$, that is, an angular wedge of width $\Delta$ is removed from the space and the remaining edges identified (see Fig. 1). This means that distant galaxies on the opposite side of a cosmic string can be gravitationally lensed to produce characteristic double images.

Cosmic strings create line-like discontinuities in the CMB signal. As the string moves across the line of sight, the CMB photons are boosted towards the observer, causing a relative CMB temperature shift across the string, given by (Gott III 1985; Kaiser \& Stebbins 1984)

$\frac{\delta T}{T}=8 \pi G \mu v_{\mathrm{s}} \gamma_{\mathrm{s}}$

where $v_{\mathrm{s}}$ is the transverse velocity of the string and $\gamma_{\mathrm{s}}=(1-$ $\left.v_{\mathrm{S}}^{2}\right)^{-1 / 2}$. This rather simple picture, however, is complicated in an

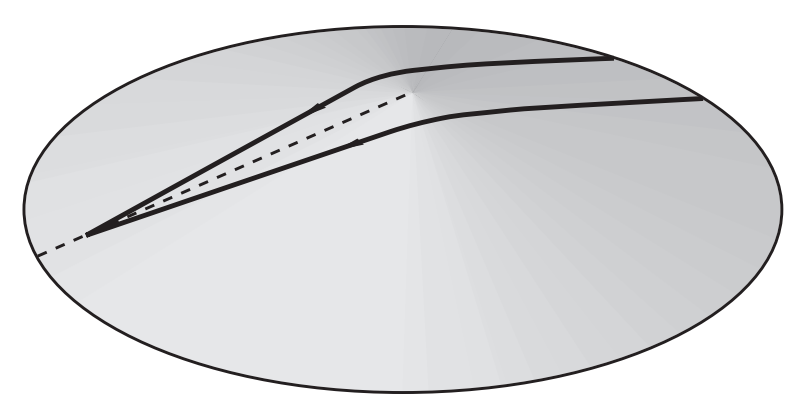

Fig. 1. The spacetime around a cosmic string is conical, as if a narrow wedge were removed from a flat sheet and the edges identified. For this reason cosmic strings can create double images of distant objects. Strings moving across the line of sight will cause line-like discontinuities in the $\mathrm{CMB}$ radiation.

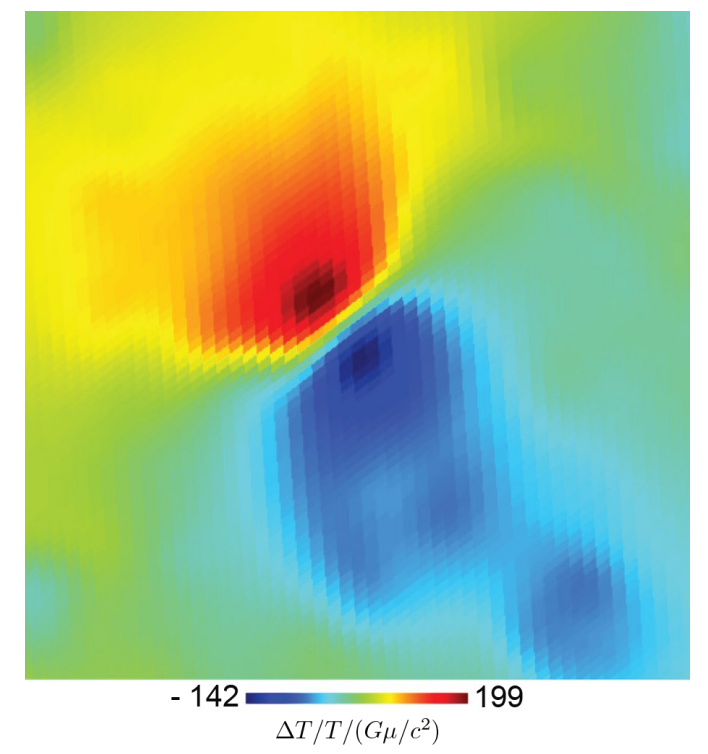

Fig. 2. Characteristic CMB temperature discontinuity created by a cosmic string. Here, the simulated Nambu-Goto string has produced a cusp, a small region on the string that approaches the speed of light, which has generated a localised CMB signal.

expanding universe with a wiggly string network and relativistic matter and radiation components. The energy-momentum tensor $T_{\mu \nu}(\boldsymbol{x}, t)$ essentially acts as a source term for the metric fluctuations that perturb the CMB photons and create temperature anisotropies. Essentially, the problem can be recast using Green's (or transfer) functions $G^{\mu v}$ that project forward the contributions of strings from early times to today:

$\frac{\Delta T}{T}\left(\hat{\boldsymbol{n}}, \boldsymbol{x}_{\mathrm{obs}}, t_{0}\right)=\int \mathrm{d}^{4} x G^{\mu v}\left(\hat{\boldsymbol{n}}, \boldsymbol{x}, \boldsymbol{x}_{\mathrm{obs}}, t, t_{0}\right) T_{\mu v}(\boldsymbol{x}, t)$,

where $\hat{\boldsymbol{n}}$ is the line-of-sight direction for photon propagation and $\boldsymbol{x}_{\mathrm{obs}}$ is the observer position. The actual quantitative solution of this problem entails a sophisticated formalism to solve the Boltzmann equation and then to follow photon propagation along the observer's line-of-sight. An example of the linelike discontinuity signal created by a cosmic string in the CMB is shown in Fig. 2. In this case, a string cusp has formed on the string, causing a strongly localised signal and reflecting the Lorentz boost factor in Eq. (8). 


\subsection{Semi-local strings}

The tight constraints on the presence of cosmic strings that we will discuss later in this paper start to put pressure on the wide class of inflation models that generate such defects (Hindmarsh 2011). The power of these constraints would be reduced if the strings could be made unstable. This is the basic motivation behind semilocal strings: a duplication of the complex scalar field $\phi$ in the Abelian-Higgs action (4), occurring naturally in a range of inflation models (Urrestilla et al. 2004; Dasgupta et al. 2004, 2007; Achucarro et al. 2006), transforms the stable cosmic strings into non-topological semilocal strings (Vachaspati \& Achucarro 1991) as the vacuum manifold becomes $S^{3}$, which is simply-connected. The existence and stability of the semilocal strings is thus a question of dynamics rather than due to the topology of the vacuum manifold. In general we do not expect to form long strings, but rather shorter string segments, as the semilocal strings can have ends. The evolution of these segments is very complicated and arises directly from the field evolution, so that it is only practicable to simulate these defects with the help of field theory (Urrestilla et al. 2008).

\subsection{Global defects}

A large alternative class of defects is due to the breaking of a global $O(N)$ symmetry (rather than a gauge symmetry as in the case of cosmic strings) of a $N$-component scalar field $\phi$. The energy density of global defects is significantly less localised than those that result from gauge symmetry breaking due to the absence of the screening by a gauge field, and there are thus long-range forces between the defects. The field self-ordering is therefore very efficient for all types of defects with $N \geq 2$, leading to a generic scaling of the defect energy density with the background energy density (see e.g., Durrer et al. 2002). For this reason global monopoles $(N=3)$ do not overclose the Universe as their local counterparts would. In this paper we study specifically the case $N=4$ called "texture", which can arise naturally in many multi-field inflation models that involve a non-zero vacuum expectation value and symmetry breaking. In this case there are no stable topological defects present, but contrary to local texture, global texture can have a non-negligible impact on the perturbations in the cosmos, with the field self-ordering leading to "unwinding events". In spite of their non-topological nature, the field evolution is closely related to the one of lowerdimensional stable global defects due to the long-range nature of the forces. This is similar to the case of the non-topological semilocal strings of the previous section, and indeed the semilocal example can be seen as an intermediate case between cosmic strings and global texture: Starting from the semilocal action, we can on the one hand revert to the cosmic string action by removing one of the complex scalar fields, and on the other hand we find the texture action if we remove the gauge field.

The normalisation of global defects is usually given in terms of the parameter $\varepsilon=8 \pi G \eta^{2} / c^{2}$ when using an action like Eq. (4) (with a second complex scalar field but without the gauge fields). However, for a simpler comparison with the cosmic string results we can recast this in terms of $G \mu / c^{2} \equiv \varepsilon / 4$ and quote limits on $G \mu / c^{2}$ also for the texture model, as in Urrestilla et al. (2008).

\subsection{CMB power spectra from cosmic defects}

The CMB power spectrum from topological defects, like strings, is more difficult to compute than the equivalent for inflationary scenarios that predict a spectrum dominated by an adiabatic component with a possible, but highly constrained, isocurvature component. In defect-based scenarios the perturbations are sourced continuously throughout the history of the Universe, as opposed to adiabatic and isocurvature modes that are the result of initial conditions. In principle this requires knowledge of the source, quantified by the unequal-time correlator (UETC) of the defect stress-energy tensor, from the time of defect formation near the GUT scale to the present day - a dynamic range of about $10^{52}$ - something that will never be possible to simulate. Fortunately, we can use the scaling assumption to extrapolate the results of simulations with substantially smaller dynamic range. This has allowed a qualitative picture to emerge of the characteristics of the power spectra from defects, though quantitative predictions differ. Here, we will focus on spectra calculated in two different ways for cosmic strings, as well as spectra from semilocal strings and texture models.

Defect-based power spectra are dominated by different physical effects across the range of angular scales. (i) On large angular scales the spectra are dominated by an integrated SachsWolfe (ISW) component due to the strings along the line-of-sight between the time of last scattering and the present day. The scaling assumption implies that this component will be close to scale invariant, although in practice it typically has a mildly blue spectrum. (ii) At intermediate scale the dominant contribution comes from anisotropies created at the time of last scattering. In contrast to the strong series of acoustic peaks created in adiabatic and isocurvature models, defects produce only a broad peak because their contributions are not coherent. (iii) At very small angular scales, the spectra are again dominated by the ISW effect because, rather than decaying exponentially due to the effects of Silk damping, there is only power-law decay with the exponent being a characteristic of the specific type of defect.

The standard lore is to treat the defect stress energy tensor, $\theta_{\mu v}$, as being covariantly conserved at first order, which is known as the "stiff approximation". In principle, this means that it is necessary to measure two independent quantities from the simulations, or model them. The other two component are then computed from the conservation equations. In practice things are a little more complicated since it is necessary to provide the UETC

$U_{\mu \nu \alpha \beta}\left(k, \tau, \tau^{\prime}\right)=\left\langle\theta_{\mu \nu}(k, \tau) \theta_{\alpha \beta}\left(k, \tau^{\prime}\right)\right\rangle$,

where $\tau$ is the conformal time and $k$ is the wavenumber. Once one has the UETC, then there two ways to proceed. The first involves creating realisations of the defect stress-energy whose power spectra are computed then averaged to give the total power spectrum. The other approach involves diagonalization of the UETC. During pure matter or radiation domination, the scaling property of defect evolution means that quantities are measured relative to the horizon scale, so that the UETC is only a function of $x=k \tau$ and $x^{\prime}=k \tau^{\prime}$. These functions $U\left(x, x^{\prime}\right)$ can be discretized and then are symmetric matrices that we can diagonalize. The resulting eigenvectors can be inserted as sources into a Boltzmann code, and the resulting $C_{\ell}$ are then summed up, weighted by the eigenvalues (Pen et al. 1997; Durrer et al. 2002). Even though the power spectrum resulting from each "eigensource" exhibits a series of acoustic peaks, the summation over many such spectra smears them out, as they are not coherent (unlike inflationary perturbations). This smearing-out explains why defect power spectra generically are smooth, as mentioned above.

There are also several methods to obtain predictions for the UETCs of cosmic strings and other topological defects. The first approach we will consider for cosmic strings is to use what has become known as the Unconnected Segment Model (USM; 


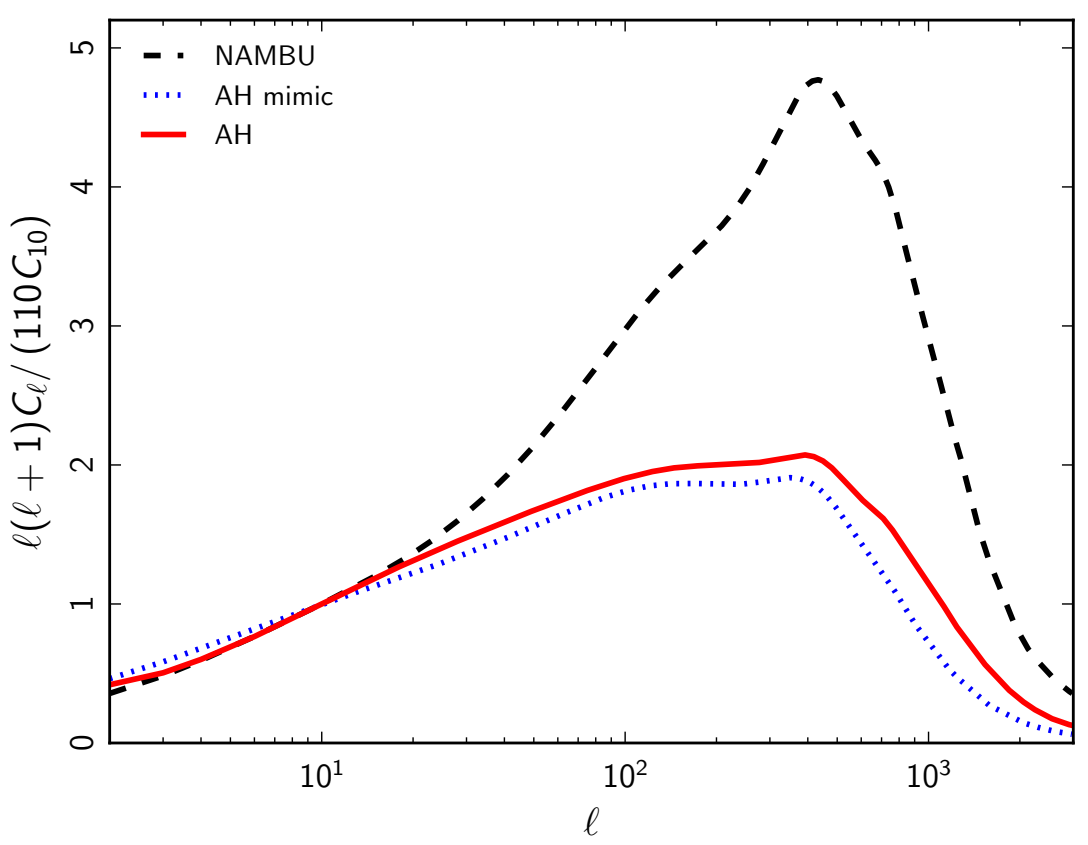

Fig. 3. Cosmic string power spectra used in this analysis: NAMBU (black dashed), AH-mimic (blue dotted) and $\mathrm{AH}$ (red solid). The spectra have been set to equal power at $\ell=10$. When normalised to $850 \mu \mathrm{K}$ at $\ell=10$ they correspond to string tensions of $G \mu / c^{2}=$ $1.17 \times 10^{-6}, 1.89 \times 10^{-6}$ and $2.04 \times 10^{-6}$ respectively. Note that the limits discussed in this paper mean that the CMB spectra presented here are less than $3 \%$ of the overall power spectrum amplitude and hence the differences observed at high $\ell$ do not have a large effect.
Albrecht et al. 1997, 1999; Pogosian \& Vachaspati 1999). In its simplest form this models the cosmic string energy momentum tensor as that of an ensemble of line segments of correlation length $\xi d_{\mathrm{H}}(t)$, moving with an rms velocity $\left\langle v^{2}\right\rangle^{1 / 2}$, where $d_{\mathrm{H}}(t)$ is the horizon distance. In addition one can take into account the effects of string "wiggles" due to small-scale structure via a coefficient, $\beta=\mu_{\mathrm{eff}} / \mu$ quantifying the ratio of the renormalised mass per unit length to the true value. The model parameters $\xi$, $\left\langle v^{2}\right\rangle^{1 / 2}$ and $\beta$ are computed from simulations. In our calculations we link the USM sources to the line-of-sight Boltzmann solver CMBACT (Pogosian \& Vachaspati 1999) to create an ensemble of realisations from which we find an averaged angular power spectrum.

There are two USM-based models that we will use in this analysis which we believe span the realistic possibilities - we note a more general approach marginalising over three string parameters is proposed in Foreman et al. (2011) (see also recent work in Avgoustidis et al. 2012). The first USM model, which we will refer to as NAMBU, is designed to model the observational consequences of simulations of cosmic string simulations performed in the Nambu-Goto approximation. In these simulations the scaling regime is different in the radiation and matter eras, with $\left(\xi,\left\langle v^{2}\right\rangle^{1 / 2} / c, \beta\right)_{\mathrm{rad}}=(0.13,0.65,1.9)$ and $\left(\xi,\left\langle v^{2}\right\rangle^{1 / 2} / c, \beta\right)_{\text {mat }}=(0.21,0.60,1.5)$ and the extrapolation between the two is modelled by using the velocity dependent onescale model (Martins \& Shellard 1996). In the second, which we will refer to as AH-mimic, we attempt to model the field theory simulations using the Abelian-Higgs model described below, with $\left(\xi,\left\langle v^{2}\right\rangle^{1 / 2} / c, \beta\right)=(0.3,0.5,1)$ independent of time.

The other approach that we will consider is to measure the UETC directly from a simulation of cosmic strings in the Abelian-Higgs model, which we will refer to as AH. The Abelian-Higgs model involves a complex scalar field $\phi$ and a gauge field $A_{\mu}$ described earlier Eq. (4), for which the dimensionless coupling constants $e$ and $\lambda$ are chosen with $\lambda=2 e^{2}$, so that the characteristic scales of the magnetic and scalar energies are equal, (see Bevis et al. (2007b,a) for further details about the model). We then simulate the evolution of the fields on a grid, starting from random initial conditions designed to mimic a phase transition, followed by a brief period of diffusive evolution, to rapidly reach a scaling solution expected to be typical of the configuration found long after the phase transition. As the simulation is performed in comoving coordinates, the string width is effectively decreasing as time passes. To enlarge the dynamical range available, we partially compensate this shrinking with an artificial string fattening. We perform runs for various values of the fattening parameter to ensure that the results are not affected by it.

During the simulations, we compute the energy-momentum tensor at regular intervals and decompose it into scalar, vector and tensor parts. We store these components once scaling is reached, and compute the UETCs by correlating them with later values of the energy-momentum tensor. UETCs from several runs are averaged, diagonalized and then fed into a modified version of the CMBEASY Boltzmann code (Doran 2005) to compute the CMB power spectra (both temperature and polarisation). The spectra used in this paper were derived from fieldtheory simulations on a $1024^{3}$ grid and used the extrapolation to sub-string scales described in Bevis et al. (2010), which are expected to be accurate at the $10 \%$ level to $\ell_{\max } \approx 4000$.

In Figs. 3 and 4 we present the spectra we will use in subsequent analysis. The higher dashed black curve is the spectrum computed using the USM for the NAMBU model, and the smaller dashed blue and solid red curves the AH-mimic model and the AH model, respectively. We should note that when normalised to the amplitude of the observed CMB anisotropies on large-scales at $\ell=10$, the three models give $G \mu / c^{2}=$ $1.17 \times 10^{-6}, 1.89 \times 10^{-6}$ and $1.9 \times 10^{-6}$ for the NAMBU, AHmimic and $\mathrm{AH}$ models, respectively. The reasons for differences between the spectra for these two approaches are discussed in Battye \& Moss (2010). Briefly, the main reasons for the differences are twofold: First, the overall normalisation, which is due to the NAMBU models having smaller values of $\xi$, more strings per horizon volume, and larger values of $\beta$, with each of the string segments being heavier, than the two AH models. Both these effects mean that a lower value of $G \mu / c^{2}$ is required to achieve the same amplitude for the anisotropies. Secondly, the enhanced peak at small angular scales, which is caused by the value of $\xi$ being smaller in the radiation era than in the matter 


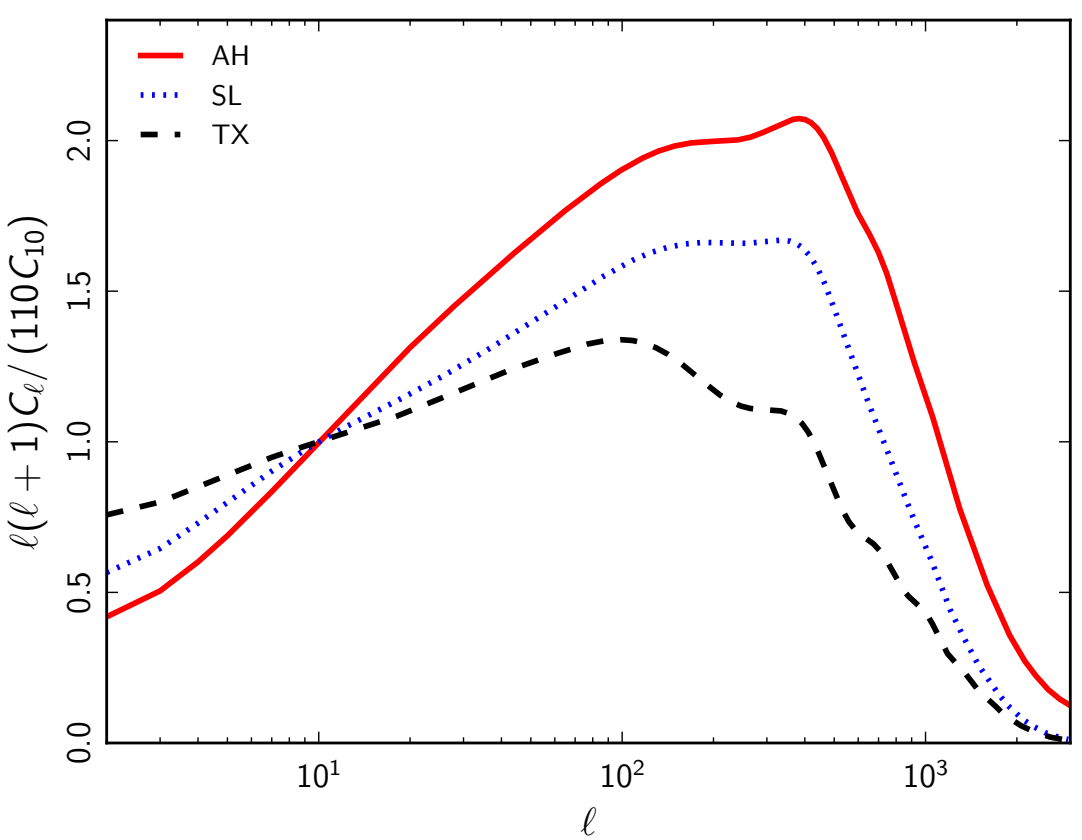

Fig. 4. Comparison between global texture (black dashed) and semilocal (blue dotted) string power spectra and the AH field theory strings (red solid), normalised to unity at $\ell=10$. As expected, the SL spectrum lies in between the TX and the AH spectra. The AH spectrum was recomputed for the Planck cosmological model with sources from Bevis et al. (2010), and the SL and TX spectra were taken from Urrestilla et al. (2008). era, meaning that there are more strings per horizon volume in the radiation era when the small-scale anisotropy is imprinted, and hence more anisotropy on those scales for a given $G \mu / c^{2}$.

The method used for the semilocal strings (denoted SL) and $O$ (4) global texture (denoted TX) is fundamentally the same as for the AH model: we simulate the field theory on a discretized grid and compute the energy-momentum tensor at regular intervals. From these snapshots we derive the UETCs by correlating the scalar, vector and tensor parts at different times. The only difference is the field-theory action being used in the simulations. In Fig. 4 we present the spectra we used for the semilocal strings and global textures, taken from Urrestilla et al. (2008). These models are also shown with the AH cosmic string model for comparison.

\subsection{Maps of $C M B$ anisotropies from cosmic strings}

In order to go further than the two-point correlation function, we have used numerical simulations of Nambu-Goto cosmic string evolution in an FLRW spacetime to generate various CMB synthetic maps. The use of simulations is crucial to produce realistic string configurations on our past light cone and have been the subject of various code development in the last twenty years (see Albrecht \& Turok 1989; Bennett \& Bouchet 1989, 1990; Allen \& Shellard 1990; Vincent et al. 1998; Moore et al. 2001; Ringeval et al. 2007; Blanco-Pillado et al. 2011). Until recently, the underlying numerical challenges have limited the resolution of the full sky maps to an angular resolution of 14' (corresponding to a HEALPix resolution of $N_{\text {side }}=256$ ) in Landriau \& Shellard $(2003,2011)$ (see also early work in Allen et al. 1996). In order to extend the applicability of these maps to the small scales probed by Planck, we have used the maps described in Ringeval \& Bouchet (2012) that have an angular resolution of $0.85^{\prime}\left(N_{\text {side }}=4096\right)$. This map is obtained by considering the ISW contribution from (9), sourced by the Nambu-Goto stress tensor, and which can be recast into the form (Stebbins \& Veeraraghavan 1995)

$$
\frac{\Delta T}{T}(\hat{\boldsymbol{n}})=-\frac{4 G \mu}{c^{2}} \int_{\boldsymbol{X} \cap \boldsymbol{x}_{\gamma}}\left[\dot{\boldsymbol{X}}-\frac{\left(\hat{\boldsymbol{n}} \cdot \boldsymbol{X}^{\prime}\right) \cdot \boldsymbol{X}^{\prime}}{1+\hat{\boldsymbol{n}} \cdot \dot{\boldsymbol{X}}}\right] \cdot \frac{X \hat{\boldsymbol{n}}-\boldsymbol{X}}{(X \hat{\boldsymbol{n}}-\boldsymbol{X})^{2}} \mathrm{~d} l .
$$

The integral is performed over all string position vectors $\boldsymbol{X}=$ $\left\{X^{i}\right\}$ intercepting our past line cone (in the transverse temporal gauge). Primes and dots denote differentiation with respect to the spatial and time-like worldsheet coordinates $\zeta^{1}$ and $\zeta^{0}$ respectively, while $\mathrm{d} l$ is the invariant string length element. Taking the limit $X \hat{\boldsymbol{n}} \rightarrow \boldsymbol{X}$ gives back the small angle and flat sky approximation used in Hindmarsh (1994); Bouchet et al. (1988); Fraisse et al. (2008). For generating the full sky map, Eq. (11) has been evaluated without any other approximation and required more than 3000 Nambu-Goto string simulations of various sizes to fill the whole comoving volume between the observer and the last scattering surface. We note that the use of different simulations does not induce any visible artefact, essentially because only the subset of strings intercepting our past light cone leaves imprints in the CMB. As a result, the probability of observing an edge remains very small. Discussions of these effects and details on the numerics can be found in Ringeval \& Bouchet (2012).

This method therefore includes all string effects from the last scattering surface till today, but does not include the Doppler contributions induced by the strings into the plasma prior to recombination. As a result, our full sky map represents the ISW contribution from strings, which is dominant at large and small scales but underestimates the signal on intermediate length scales where recombination effects on the surface of last scattering dominate (see the discussion in Sect. 2.4). This can be seen directly by making a qualitative comparison between the ISW string power spectrum, obtained from the simulated maps and shown in Fig. 5, with the full CMB string power spectrum predicted for Nambu strings shown in Fig. 3 (dashed line). We therefore expect the string searches based on the simulated maps to be less constraining than those using the power spectrum, though certainly robust as any line-like gravitating object should generate such a signal.

Calibration and training for the non-Gaussian searches of Sect. 4 have required the generation of new full sky and statistically independent cosmic string maps. The numerical challenges underlying the $N_{\text {side }}=4096$ map (Ringeval \& Bouchet 2012) are such that it was numerically too expensive to create another one of the same kind. At this resolution, the computations typically require $800000 \mathrm{cpu}$-hours, so we have chosen 


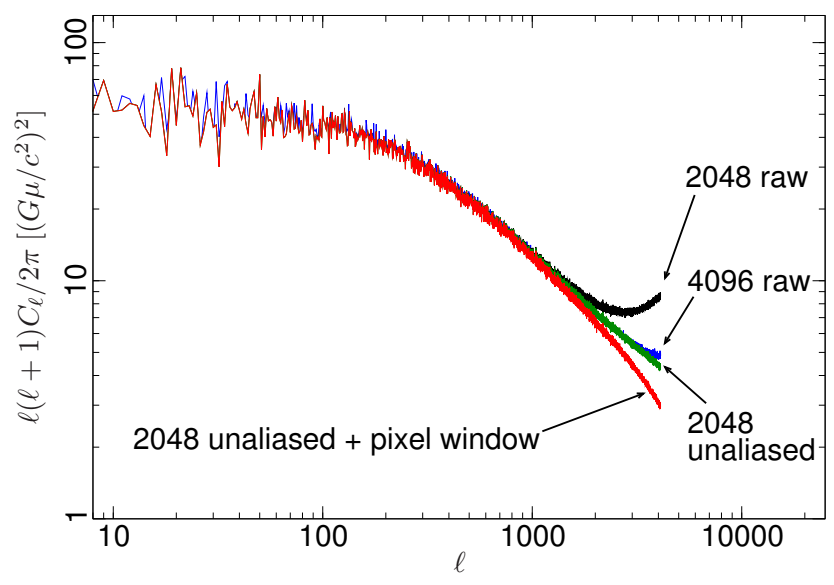

Fig. 5. Integrated Sachs-Wolfe angular power spectra extracted from the full sky cosmic string maps at different resolutions (labelled by $N_{\text {side }}$ ), with or without applying the anti-aliasing procedure (see text). The antialiasing filtering gives back the correct power up to $\ell_{\max } \lesssim 2 N_{\text {side }}$.

to generate three new maps at a lower resolution of $1.7^{\prime}$, i.e., $N_{\text {side }}=2048$. Unfortunately, at this lower resolution, the simulated string maps, hereafter referred to as raw maps, exhibit a strong aliasing at small scales that could have induced spurious systematics even after convolution with the Planck beam. This aliasing concerns pixel-sized structures and comes from the method used to numerically evaluate Eq. (11). In order to save computing time, the signal associated with each pixel is only computed at the centroid direction $\hat{\boldsymbol{n}}$. This has the effect of including some extra power associated with string small-scale structure that is below the pixel angular size, thereby aliasing power into the map. In order to address this problem, we have used semi-analytical methods to design an optimal anti-aliasing filter, both in harmonic space and in real space. As discussed in Fraisse et al. (2008); Bevis et al. (2010), the small scale angular temperature power spectrum slowly decays as a power law $\ell^{-p}$ such that any deviations from this behaviour can only come from the aliasing. For each $N_{\text {side }}=2048$ raw map, we have performed a multi-parameter fit of the power spectrum, and of the one-point distribution function, to extract, and then removes, its small scale aliasing contribution. In order to validate the procedure, we have checked that the power spectrum of each of the filtered maps matches the one associated of the raw $N_{\text {side }}=4096$ map, the latter being also being affected but at half the scale. In Fig. 5, we have plotted the power spectra of one of the $N_{\text {side }}=2048$ maps before and after convolution with our anti-aliasing filter. As expected, it matches with the one extracted from the $N_{\text {side }}=4096$ map (here truncated at $\ell=4096$ ). We note that the string bispectra reconstructed from the antialiased $N_{\text {side }}=2048$ maps and the $N_{\text {side }}=4096$ maps are essentially identical, as discussed in Sect. 4.2.2 and shown in Fig. 11. Finally, in order to include the effects associated with the HEALPix pixelization scheme, the anti-aliased maps have been convolved with the HEALPix pixel window function before being used for further processing.

In total, this method has provided four theoretical full sky string maps that have been used in the string searches we will discuss in Sect. 4. As an illustration, we have represented in Fig. 6, one of the filtered string map after convolution by a Gaussian beam of $F W H M=5^{\prime}$. The colour scale traces the relative temperature anisotropies $\Delta T / T$, divided by the string tension $G \mu / c^{2}$. The anisotropy patterns may look Gaussian at first because most of the string signatures show up on the smallest length scales. In Fig. 7, we have plotted a gnomic projection representing a field of view of $20^{\circ}$, in which the temperature steps are now clearly apparent. The right panel of Fig. 7 represents the magnitude of the spherical gradient, which enhances the steps.

Finally, in order to provide a much larger statistical sample beyond only four string realisations, we have also produced a collection of 1000 small angle patches $\left(7.2^{\circ}\right)$ of the CMB sky derived in the flat sky approximation (Stebbins 1988; Hindmarsh 1994; Stebbins \& Veeraraghavan 1995; Bouchet et al. 1988; Fraisse et al. 2008). Although the large-scale correlations are lost, these maps have been shown to accurately reproduce various analytically expected non-Gaussian string effects such as the one-point and higher $n$-points functions by Takahashi et al. (2009), Hindmarsh et al. (2009), Hindmarsh et al. (2010), Regan \& Shellard (2010), Yamauchi et al. (2010b), Yamauchi et al. (2010a), Ringeval (2010).

\section{Power spectrum constraints on cosmic strings and other topological defects}

In order to compute constraints on cosmic string scenarios we just add the angular power spectrum to that for an simple adiabatic model - which assumes that they are uncorrelated - with the fraction of the spectrum contributed by cosmic strings being $f_{10}$ at $\ell=10$. This parameter is then added as an extra parameter to the standard six parameter fit using COSMOMC and the Planck likelihood described in Planck Collaboration XV (2014). We use a Flat $\Lambda C D M$ cosmology defined through the physical densities of baryons, $\Omega_{\mathrm{b}} h^{2}$, and cold dark matter, $\Omega_{\mathrm{c}} h^{2}$, the acoustic scale, $\theta_{\mathrm{M} C}$, the amplitude, $A_{\mathrm{s}}$ and spectral index, $n_{\mathrm{s}}$ of density fluctuations and the optical depth to reionization $\tau$. The Hubble constant is a derived parameter and is given by $H_{0}=100 \mathrm{hm} \mathrm{s}^{-1} \mathrm{Mpc}^{-1}$. We use the same priors on the cosmological and nuisance parameters as are used in Planck Collaboration XVI (2014) and use WMAP polarisation data to help fix $\tau$. In addition to just using the Planck data, we have also added high- $\ell$ CMB data from SPT and ACT to obtain stronger constraint(Sievers et al. 2013; Hou et al. 2014).

For the USM-based models we use the approach used in Battye et al. (2006) and Battye \& Moss (2010). We find that the constraints on the standard six parameters are not significantly affected by the inclusion of the extra string parameter and that there are no significant correlations with other parameters (see Table 3). For the case of Planck data only and using the NAMBU model we find that $G \mu / c^{2}<1.5 \times 10^{-7}$ and $f_{10}<0.015$, whereas for the AH-mimic model we find that $G \mu / c^{2}<3.6 \times 10^{-7}$ and $f_{10}<0.033$, with all the upper limits being at $95 \%$ confidence level. The 1D marginalised likelihoods for $f_{10}$ are presented in the upper panels of Fig. 8. The differences between the upper limits for the NAMBU and $\mathrm{AH}-$ mimic models is compatible with those seen previously using WMAP 7-year and SDSS data (Battye \& Moss 2010). The upper limits from this version of the Planck likelihood are better than those computed from WMAP7+SPT (Dvorkin et al. 2011) and WMAP7+ACT (Dunkley et al. 2011) and are significantly better than those from WMAP7+SDSS (Battye \& Moss 2010). Based on the Planck "Blue Book" values for noise levels we predicted (Battye et al. 2008) a limit of $G \mu / c^{2}<6 \times 10^{-8}$, while the present limit is about a factor of two worse than this. The main reason for this is that the projected limit ignored the need for nuisance parameters to model high $\ell$ foregrounds and that not all the frequency channels have been used. The corresponding limits for the AH model are $f_{10}<0.028$ and $G \mu / c^{2}<3.2 \times 10^{-7}$. 


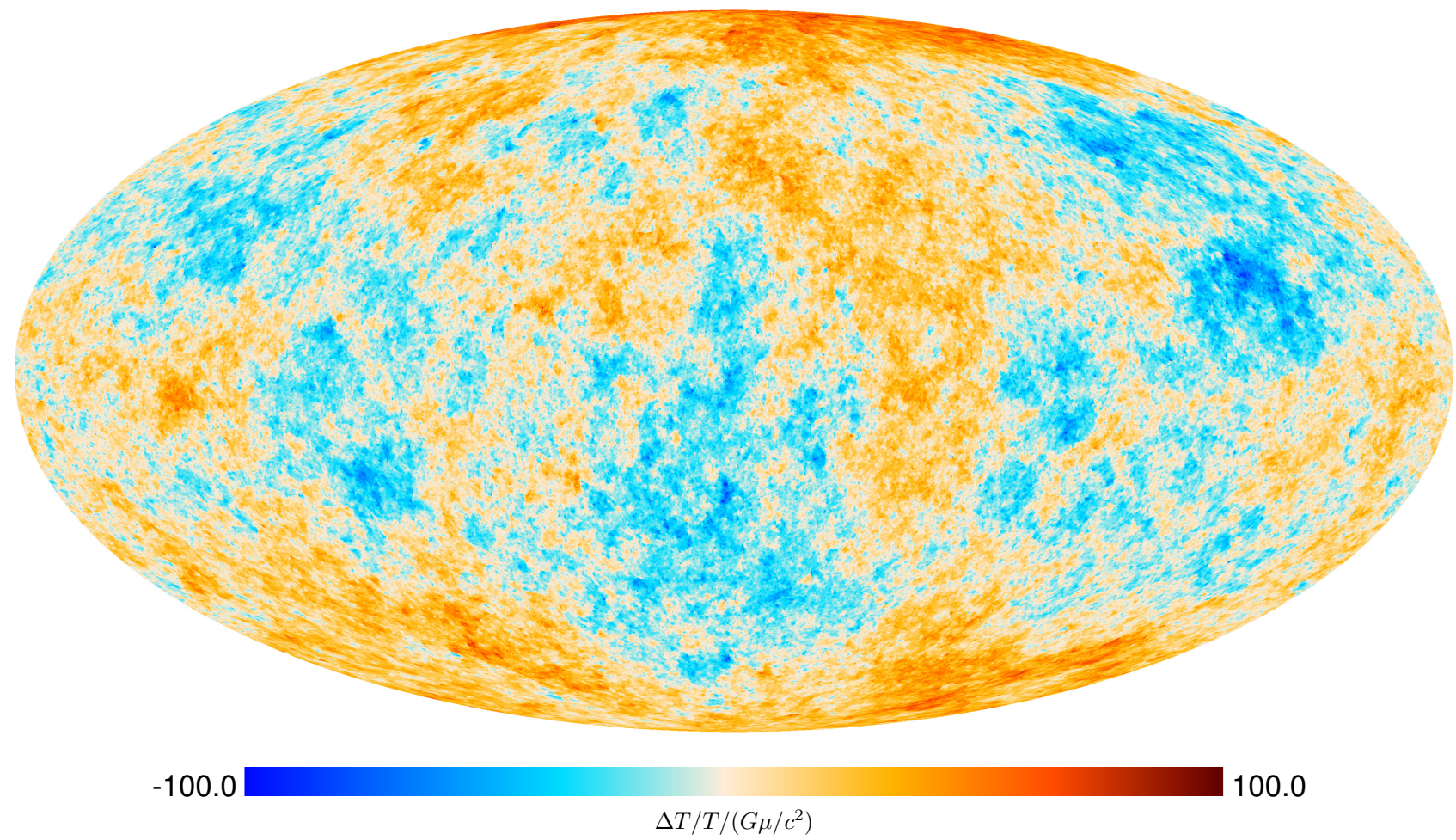

Fig. 6. All sky Mollweide projection of the simulated cosmic strings CMB sky after convolution by a Gaussian beam of $5^{\prime}$ resolution. The colour scale indicates the range of $(\Delta T / T) /\left(G \mu / c^{2}\right)$ fluctuations.
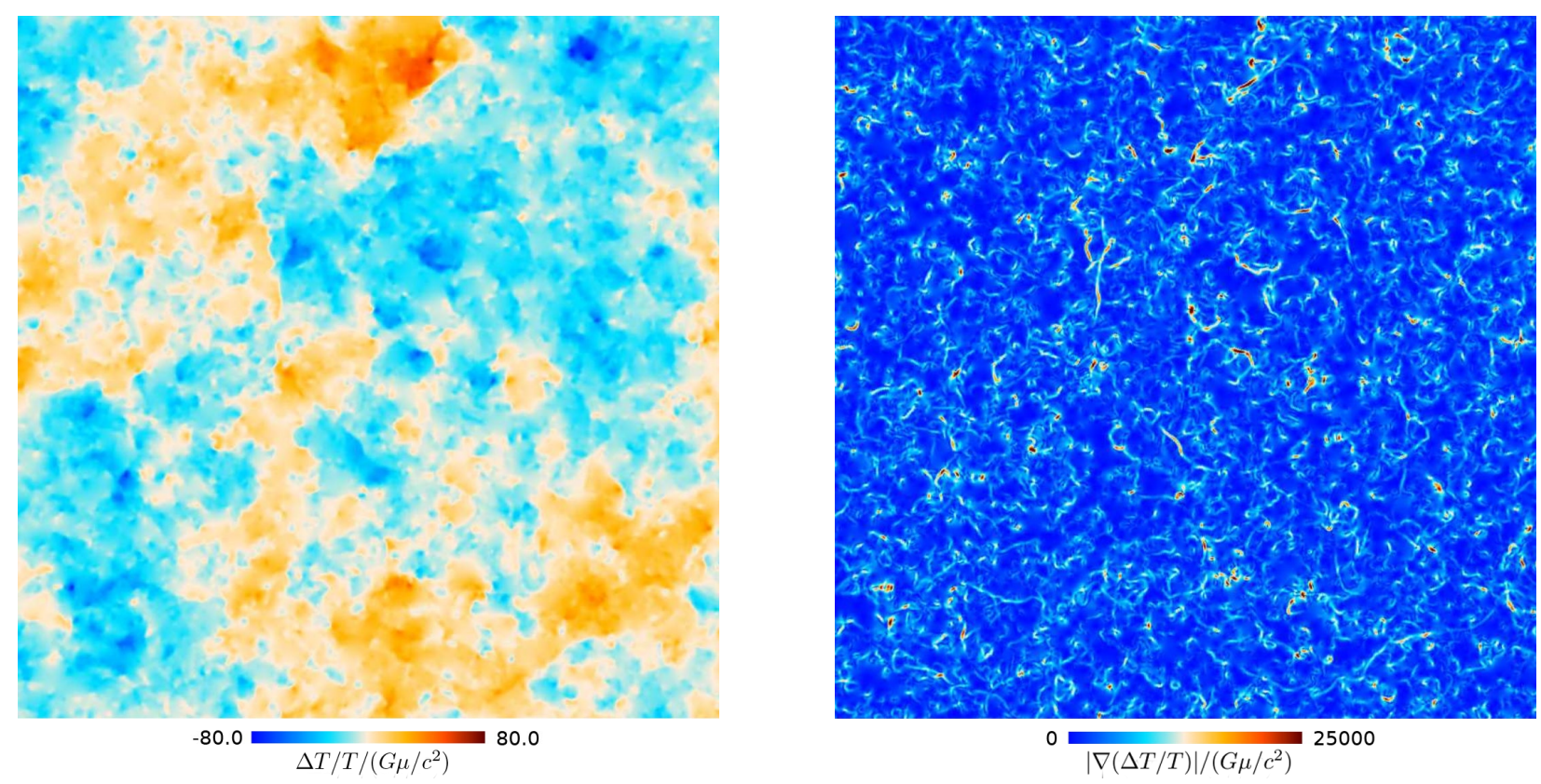

Fig. 7. A $20^{\circ}$ gnomic projection patch extracted from the full sky map and zooming into string induced temperature steps (see Fig. 6). Applying the spherical gradient magnitude operator enhances the temperature steps, and thus the string locations, even more (right).

There is now very little degeneracy between the $f_{10}$ and $n_{\mathrm{S}}$ parameters, something that was not the case for WMAP alone (Battye et al. 2006; Bevis et al. 2008; Urrestilla et al. 2011). This has implication for supersymmetric hybrid inflation models as discussed in Battye et al. (2010) that typically require $n_{\mathrm{S}}>0.98$. The simplest versions of these models appear to be ruled out. The strongest correlation using the NAMBU and AH mimic models is between $f_{10}$ and $\Omega_{\mathrm{b}} h^{2}$ as illustrated in Fig. 10. For the AH model the correlation between $f_{10}$ and the amplitude of primordial perturbations, $A_{\mathrm{s}}$, is of a comparable magnitude, for all other parameters (including the nuisance parameters) the correlations are even less important. In addition, we find in agreement with Lizarraga et al. (2012), that there are significant correlations between the amount of strings $f_{10}$ in the $\mathrm{AH}$ model and the number of relativistic degrees of freedom $N_{\text {eff }}$ as well as between $f_{10}$ and the primordial helium abundance $Y_{\mathrm{He}}$. We leave a detailed study of these correlations to later work. 
Table 3. Constraints on the fitted cosmological parameters in the case of Planck alone for the cosmic strings models (NAMBU, AH-mimic and $\mathrm{AH})$, semilocal strings (SL) and texture (TX).

\begin{tabular}{lcccccc}
\hline \hline \multicolumn{1}{c}{ Parameter } & No defects & NAMBU & AH-mimic & AH & SL & TX \\
\hline$\Omega_{\mathrm{b}} h^{2} \ldots \ldots \ldots \ldots \ldots$ & $0.0221 \pm 0.0003$ & $0.0223 \pm 0.0003$ & $0.0223 \pm 0.0003$ & $0.0223 \pm 0.0003$ & $0.0223 \pm 0.0003$ & $0.0223 \pm 0.0003$ \\
$\Omega_{\mathrm{c}} h^{2} \ldots \ldots \ldots \ldots \ldots$ & $0.119 \pm 0.003$ & $0.119 \pm 0.003$ & $0.119 \pm 0.003$ & $0.119 \pm 0.003$ & $0.119 \pm 0.003$ & $0.119 \pm 0.003$ \\
$\theta_{\mathrm{M} C} \ldots \ldots \ldots \ldots$ & $1.0413 \pm 0.0006$ & $1.0415 \pm 0.0006$ & $1.0415 \pm 0.0006$ & $1.0415 \pm 0.0006$ & $1.0415 \pm 0.0006$ & $1.0415 \pm 0.0006$ \\
$\tau \ldots \ldots \ldots \ldots$ & $0.089 \pm 0.013$ & $0.089 \pm 0.013$ & $0.090 \pm 0.013$ & $0.090 \pm 0.013$ & $0.090 \pm 0.013$ & $0.088 \pm 0.014$ \\
$\log \left(10^{10} A_{\mathrm{s}}\right) \ldots \ldots \ldots$ & $3.089 \pm 0.025$ & $3.080 \pm 0.027$ & $3.080 \pm 0.026$ & $3.081 \pm 0.025$ & $3.081 \pm 0.025$ & $3.078 \pm 0.028$ \\
$n_{\mathrm{s}} \ldots \ldots \ldots \ldots$ & $0.960 \pm 0.007$ & $0.961 \pm 0.007$ & $0.963 \pm 0.008$ & $0.963 \pm 0.008$ & $0.964 \pm 0.007$ & $0.965 \pm 0.008$ \\
$H_{0} \ldots \ldots \ldots \ldots \ldots$ & $67.3 \pm 1.2$ & $68.4 \pm 1.3$ & $68.3 \pm 1.2$ & $68.3 \pm 1.3$ & $68.2 \pm 1.2$ & $68.3 \pm 1.2$ \\
$G \mu / c^{2} \ldots \ldots \ldots \ldots$ & - & $<1.5 \times 10^{-7}$ & $<3.6 \times 10^{-7}$ & $<3.2 \times 10^{-7}$ & $<1.10 \times 10^{-6}$ & $<1.06 \times 10^{-6}$ \\
$f_{10} \ldots \ldots \ldots \ldots \ldots$ & - & $<0.015$ & $<0.033$ & $<0.028$ & $<0.043$ & $<0.055$ \\
\hline
\end{tabular}

Notes. It is clear from this that the fitted parameters are not significantly affected by the inclusion of defects (see also Fig. 10 which shows the strongest correlation, $f_{10}-\Omega_{\mathrm{b}} h^{2}$ ).
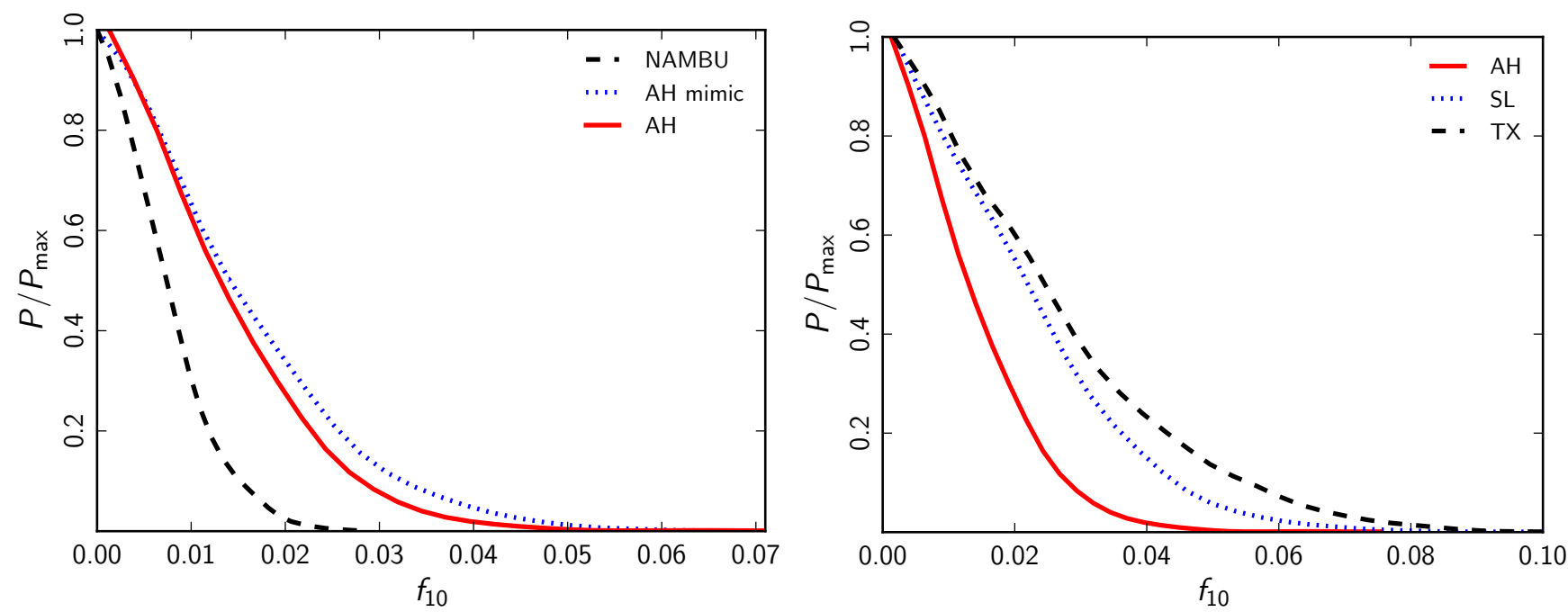

Fig. 8. Marginalised constraints on $f_{10}$ for topological defects from Planck data plus polarisation from WMAP (Planck+WP). The left panel show constraints on cosmic strings, with NAMBU in black dashed, AH-mimic in blue dotted and AH in red solid. The right panel show the constraints on SL (blue dotted) and TX (black dashed) compared to AH (again solid red).
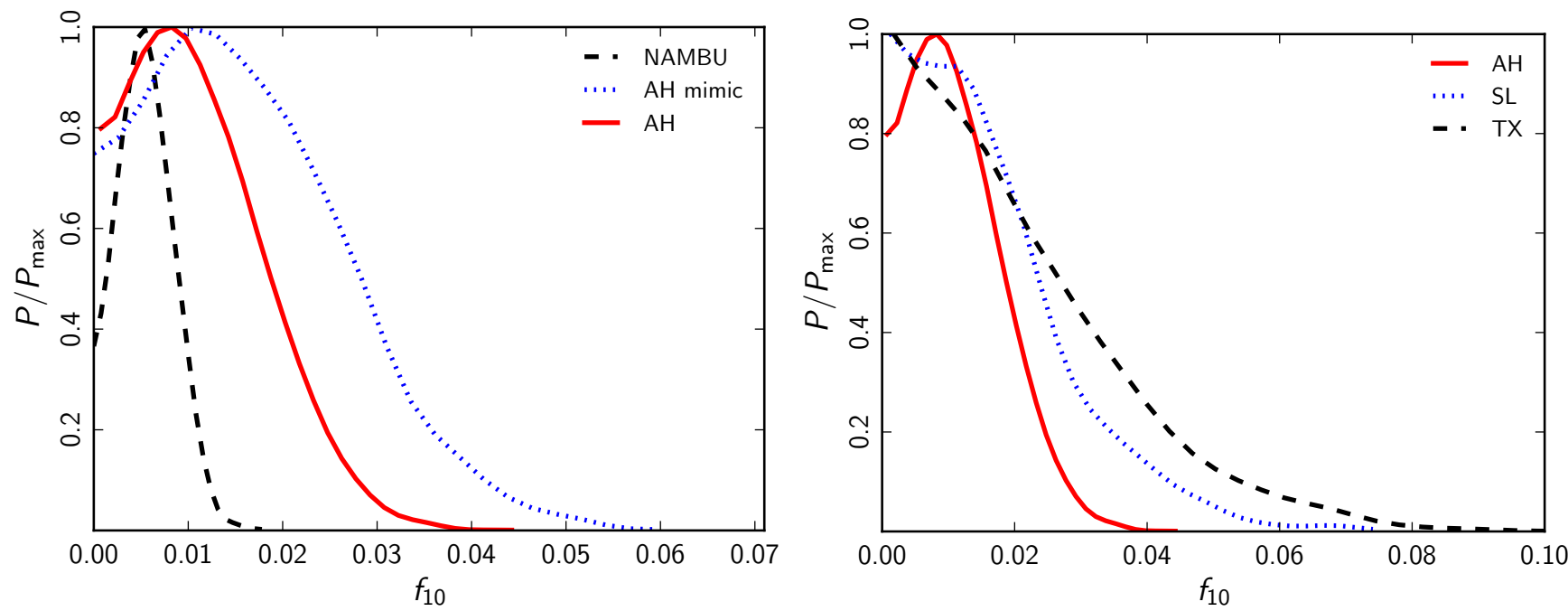

Fig. 9. Marginalised constraints on $f_{10}$ for topological defects with high $\ell$ CMB data from SPT and ACT added to the Planck + WP constraints data (compare with constraints shown in Fig. 8). The left panel show constraints on cosmic strings, with NAMBU in black dashed, AH-mimic in blue dotted and AH in red solid. The right panel show the constraints on SL (blue dotted) and TX (black dashed) compared to AH (solid red). 


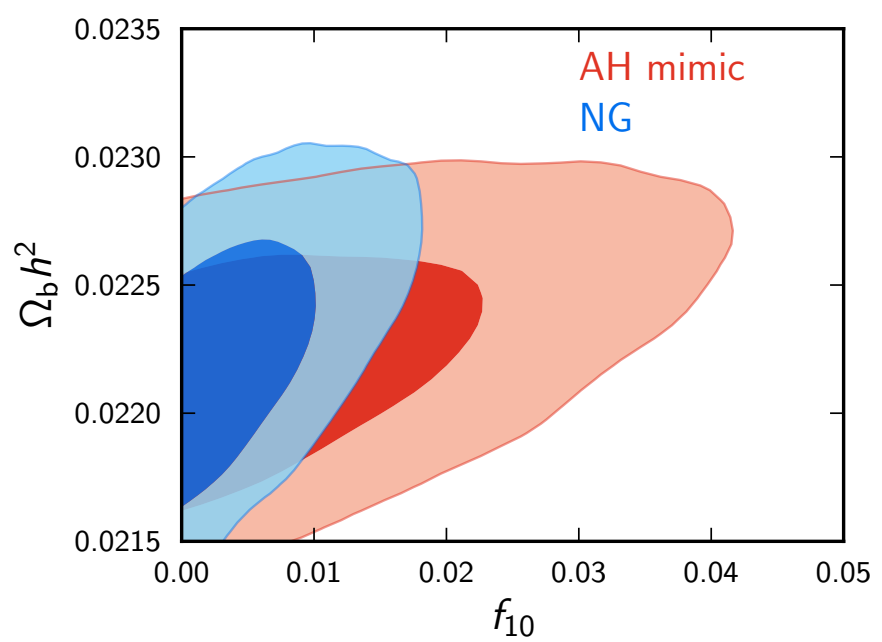

Fig. 10. Marginalised likelihoods in the $f_{10}-\Omega_{\mathrm{b}} h^{2}$ plane for the NAMBU model in blue and the AH mimic model in red using Planck +WP. This is the strongest correlation with any of the standard cosmological parameters.

In Fig. 8 we also present the 1D marginalised likelihoods for the texture and semilocal string models (compared to the $\mathrm{AH}$ field theory strings). The resulting constraints on the $f_{10}$ parameter are given in Table 2 as well. For the conversion into constraints on $G \mu / c^{2}$ we have that for semilocal strings $G \mu_{10} / c^{2}=$ $5.3 \times 10^{-6}$ and for global texture $G \mu_{10} / c^{2}=4.5 \times 10^{-6}$, cf. Urrestilla et al. (2008). We notice that, as expected for a fixed $G \mu$, semilocal strings lead to significantly less anisotropies than cosmic strings (a factor of about 8 in the $C_{\ell}$ ), and texture are similar to the semilocal strings. We thus expect significantly weaker constraints on $G \mu$ for the SL and TX models, especially since in addition the constraints on $f_{10}$ for these models are weaker. Indeed we find a $95 \%$ limit of $G \mu / c^{2}<1.10 \times 10^{-6}$ for semilocal strings and $G \mu / c^{2}<1.06 \times 10^{-6}$ for global textures.

\section{Non-Gaussian searches for cosmic strings}

Cosmic strings and other topological defects generically create non-Gaussian signatures in the cosmic microwave sky, counterparts of their inevitable impact on the CMB power spectrum. This is a critical test of differentiating defects from simple inflation, while offering the prospect of direct detection. Searches for these non-Gaussian defect signatures are important for two key reasons: on the one hand, constraints from the CMB power spectrum can be susceptible to degeneracies with cosmological parameters in the standard concordance model; on the other hand, any apparent defect detection in the power spectrum should have a well-defined prediction in higher-order correlators or other non-Gaussian signals, and vice versa. Non-Gaussian tests can also be used to distinguish cosmic defects from residual foregrounds or systematic contributions. Below we will present results from NG tests that seek strings in multipole space (bispectrum) and in real space (Minkowski functionals), as well as hybrid methods (wavelets).

\subsection{Foregrounds, systematics and validation}

It is well-known that the microwave sky contains not only the CMB signal but also emission from different astrophysical contaminants. In particular, point source emission is expected to be a special cause of confusion for cosmic defects, notably those with high resolution signatures, such as cosmic strings. In addition, systematic effects may also be present in the maps at a certain level. Therefore, before claiming a cosmological origin of a given detection, alternative extrinsic sources should be investigated and discarded. This can be done by performing a number of consistency checks in the data, most of which are common to the other non-Gaussianity papers, where they are discussed in greater detail. Here, we provide a brief summary of the main issues.

Foreground-cleaned CMB maps are provided using four different component separation techniques (for further details, see Planck Collaboration XII 2014): SMICA (semi-blind approach); NILC (internal linear combination in needlet space);SEVEM (internal template fitting); and Commander/Ruler (C-R, parametric method). These four foreground-separation methods are complimentary in that some work in pixel space while others in harmonic space (or a combination with wavelets). They were tested using the most realistic Planck simulations available, i.e. the Full Focal Plane (FFP) version 6, including demonstration that they preserved non-Gaussian signals as we will discuss below. In determining the robustness of a particular constraint, we note that it should be replicated with at least two different cleaned CMB maps. The adoption of different masks that exclude different regions of the sky (ranging from more aggressive to more conservative) has also been used to test the stability of non-Gaussian estimators. Further tests entail the use of cleaned maps at different frequencies (for instance, those provided by the SEVEM foreground separation technique). A given detection should be consistent at all frequencies, since the behaviour of contaminants and systematic effects will, in general, vary with frequency. A further test is the study of noise maps constructed from the difference between two Planck maps (either at the same or at different frequencies) smoothed to the same resolution. These maps will not contain the CMB signal and, therefore, any NG detection should vanish on them. The opposite would indicate that the claimed result is due to foreground residuals or to the presence of systematic effects.

The methodologies described below were tested using several approaches, passing through the non-Gaussian validation suite, involving realistic FFP6 simulations, and culminating in a series of Planck String Challenges instituted for this purpose. The non-Gaussianity validation tests are described in detail elsewhere (see Planck Collaboration XXIV 2014) and the realistic FFP6 simulations also (see Planck Collaboration 2013), nevertheless we include a summary here because of their importance also for the validation of cosmic string searches. A set of 96 non-Gaussian maps, with given local, equilateral and orthogonal signals, were created using the methods described in Fergusson et al. (2010a), to which was added coloured and anisotropic noise as specified from the SMICA cleaned map. All NG estimators, notably the modal bispectrum method, were required to identify these (unknown) input signals within the expected precision. Realistic Planck FFP6 simulations, including an unknown non-Gaussian signal, were then used for the next level of validation. These simulations are intended to provide a complete description of the full Planck mission for both HFI and LFI instruments, including the best current estimates of foreground templates (CIB, CO, free-free, thermal dust etc.), the lensed primordial CMB signal (using lensPIX), timestream effects, such as satellite pointing, focal plane geometries, and ring-by-ring detector noise spectra, and finally the simulated time-ordered data was synthesised together using the Planck map-making pipeline at each frequency. The FFP6 maps were then passed through the four component-separation pipelines and NG estimators (both 
modal and MF) were able to detect the (unknown) $f_{\mathrm{nl}}=20.4$ signal (see Planck Collaboration XXIV 2014). We note the caveat that the FFP simulations continue to evolve and improve by incorporating systematic effects more completely and realistically, including "de-glitching" and polarisation effects in the near future. Given the available time and resources, the FFP6 simulation set (on which $15 \mathrm{M}$ core hours were expended) represents the current state-of-the-art for this first Planck defects analysis, as well as other non-Gaussian analysis.

Similar to the non-Gaussian validation suite, the Planck String Challenges were blind tests employing postrecombination string simulation maps, with an unknown $G \mu / c^{2}$ (as described in Sect. 2.5), co-added to a Gaussian CMB map (created using WMAP7 cosmological parameters). The effect of a 5 arcmin beam was added, together with anisotropic SEVEM noise, and the same mask was employed as for the non-Gaussian analysis $\left(f_{\text {sky }}=0.73\right)$ For calibrating the string searches, a further three string simulations were also provided (without noise). A further $1000 \Lambda \mathrm{CDM}$ Gaussian maps with SEVEM noise and beam effects were also provided for validation and calculating linear correction terms. The second challenge incorporated improvements in the coloured SEVEM noise model and lensed Gaussian CMB maps. The aim was to determine the sensitivity of the proposed non-Gaussian tests and to see if the $G \mu / c^{2}$ in the challenge map could be measured accurately. The results from these challenges were an important part of the validation for each of the methods described below. In addition, Gaussian FFP6 simulations pipelines for each of the component separation methods were available for determining whether expected foreground residuals were correlated with the string signal.

\subsection{Cosmic string bispectrum}

\subsubsection{Modal bispectrum methods}

The CMB bispectrum is the three point correlator of the $a_{l m}$ coefficients, $B_{m_{1} m_{2} m_{3}}^{\ell_{1} \ell_{3}}=a_{\ell_{1} m_{1}} a_{\ell_{2} m_{2}} a_{\ell_{3} m_{3}}$. For the purposes of a search for cosmic strings we assume the network cumulatively creates a statistically isotropic signal, that is, we can employ the angleaveraged reduced bispectrum $b_{\ell_{1} \ell_{2} \ell_{3}}$, defined by

$b_{\ell_{1} \ell_{2} \ell_{3}}=\sum_{m_{i}} h_{\ell_{1} \ell_{2} \ell_{3}}^{-2} \mathcal{G}_{m_{1} m_{2} m_{3}}^{\ell_{1} \ell_{2} \ell_{3}} B_{m_{1} m_{2} m_{3}}^{\ell_{1} \ell_{2} \ell_{3}}$

where $h_{\ell_{1} \ell_{2} \ell_{3}}$ is a weakly scale-dependent geometrical factor and $\mathcal{G}_{m_{1} m_{2} m_{3}}^{l_{1} l_{2} l_{3}}$ is the well-known Gaunt integral over three $Y_{\ell m} \mathrm{~S}$ that can be expressed in terms of Wigner- $3 j$ symbols. The CMB bispectrum $b_{\ell_{1} \ell_{2} \ell_{3}}$ is defined on a tetrahedral domain of multipole triples $\left\{\ell_{1} \ell_{2} \ell_{3}\right\}$ satisfying both a triangle condition and $\ell<\ell_{\max }$ set by the experimental resolution. When seeking the string bispectrum $b_{\ell_{1} \ell_{2} \ell_{3}}^{\text {string }}$ in the Planck data, we employ the following estimator to find or limit its amplitude:

$\mathcal{E}=\frac{1}{\tilde{N}^{2}} \sum_{l_{i} m_{i}} \frac{\mathcal{G}_{m_{1} m_{2} m_{3}}^{\ell_{1} \ell_{2} \ell_{3}} b_{\ell_{1} \ell_{2} \ell_{3}}^{\text {string }}}{\tilde{C}_{\ell_{1}} \tilde{C}_{\ell_{2}} \tilde{C}_{\ell_{3}}} a_{\ell_{1} m_{1}} a_{\ell_{2} m_{2}} a_{\ell_{3} m_{3}}$,

where we assume a nearly diagonal covariance matrix $C_{\ell_{1} m_{1}, \ell_{2} m_{2}} \approx C_{\ell} \delta_{\ell_{1} \ell_{2}} \delta_{m_{1}-m_{2}}$ and we modify $C_{\ell}$ and $b_{\ell_{1} \ell_{2} \ell_{3}}$ appropriately to incorporate instrument beam and noise effects, as well as a cut-sky. To simplify Eq. (13), we have ignored the "linear term" (which is included in the analysis). A much more extensive introduction to bispectrum estimation can be found in Planck Collaboration XXIV (2014).
A key step in observational searches for non-separable bispectra, such as those induced by cosmic strings (denoted by $b_{\ell_{1} \ell_{2} \ell_{3}}^{\text {string }}$ ), is to expand it into separable modes (Fergusson \& Shellard 2009; Fergusson et al. 2010a) taking the signal-to-noise-weighted form:

$\frac{b_{\ell_{1} \ell_{2} \ell_{3}}^{\text {string }}}{\sqrt{C_{\ell_{1}} C_{\ell_{2}} C_{\ell_{3}}}}=\sum_{n} \alpha_{n}^{Q} Q_{n}\left(\ell_{1}, \ell_{2}, \ell_{3}\right)$,

where the modes $Q_{n}\left(\ell_{1}, \ell_{2}, \ell_{3}\right)=\frac{1}{6}\left[\bar{q}_{p}\left(l_{1}\right) \bar{q}_{r}\left(l_{2}\right) \bar{q}_{s}\left(l_{3}\right)+\right.$ perms. $]$ are constructed from symmetrized products (the $n$ label a distance-ordering for the triples $\{p r s\})$. The product basis functions $Q_{n}\left(\ell_{1}, \ell_{2}, \ell_{3}\right)$ are not in general orthogonal, so it is very useful to construct a related set of orthonormal mode functions $R_{n}\left(\ell_{1}, \ell_{2}, \ell_{3}\right)$ such that $\left\langle R_{n}, R_{p}\right\rangle=\delta_{n p}$. Substituting the separable mode expansion (14) reduces the estimator (13) to the simple form

$\mathcal{E}=\frac{1}{N^{2}} \sum_{n} \alpha_{n}^{Q} \beta_{n}^{Q}$,

where the $\beta_{n}^{Q}$ coefficients are found by integrating products of three Planck maps filtered using the basis function $\alpha_{n}^{Q}$ (an efficient product with each map multiplied by the separable $q_{r}(\ell)$ ). We can validate this estimator by using the modal methodology to create CMB map realisations for cosmic strings from the predicted $\beta_{n}^{Q}$ with a given $G \mu / c^{2}$ (see Fergusson et al. 2010a). It is easy to show that the expectation value for $\beta_{n}^{R}$ for such an ensemble of maps in the orthogonal basis should be

$\left\langle\beta_{n}^{R}\right\rangle=\alpha_{n}^{R}$.

Alternatively, we can exploit this fact by reconstructing the $\alpha_{n}^{R}$ from given $\mathrm{CMB}$ map realisations created directly from string simulations, an approach we will adopt here.

\subsubsection{Post-recombination string bispectrum}

In order to estimate the string bispectrum at Planck resolution we employed the modal reconstruction method Eq. (16) on the post-recombination string simulations described in Sect 2.5. These string maps include the accumulated line-like discontinuities induced by the string network on CMB photons propagating from the surface of last scattering to the present day. This work does not include recombination physics, that is, contributions from the surface of last scattering that will increase the string anisotropy signal substantially. As discussed previously, there are four full-sky maps of two different resolutions, which were provided for the purpose of calibrating Planck searches for cosmic strings (Ringeval \& Bouchet 2012). For the modal analysis, we have adopted the hybrid polynomial basis augmented with a local shape mode (in total with $n_{\max }=600$ modes), as well as a hybrid Fourier basis $\left(n_{\max }=300\right)$, which are both described in Planck Collaboration XXIV (2014).

To extract the string bispectrum in a Planck-realistic context, we chose a fairly high non-Gaussian signal with $G \mu / c^{2}=1 \times$ $10^{-6}$. As for the Planck String Challenges, described in Sect. 4.1, the normalised string maps were added to noise maps generated by the component separation pipelines of SMICA, NILC and SEVEM, creating twelve sets of 200 simulated string realisations. Each of these maps was then filtered using the modal estimator to find the $\beta_{n}^{R}$ coefficients appropriate for each componentseparation method. After averaging each set of modal coefficients $\alpha_{n}^{R}=\left\langle\beta_{n}^{R}\right\rangle$ over the different (unmasked) noise realisations, we found remarkable consistency between the estimated 

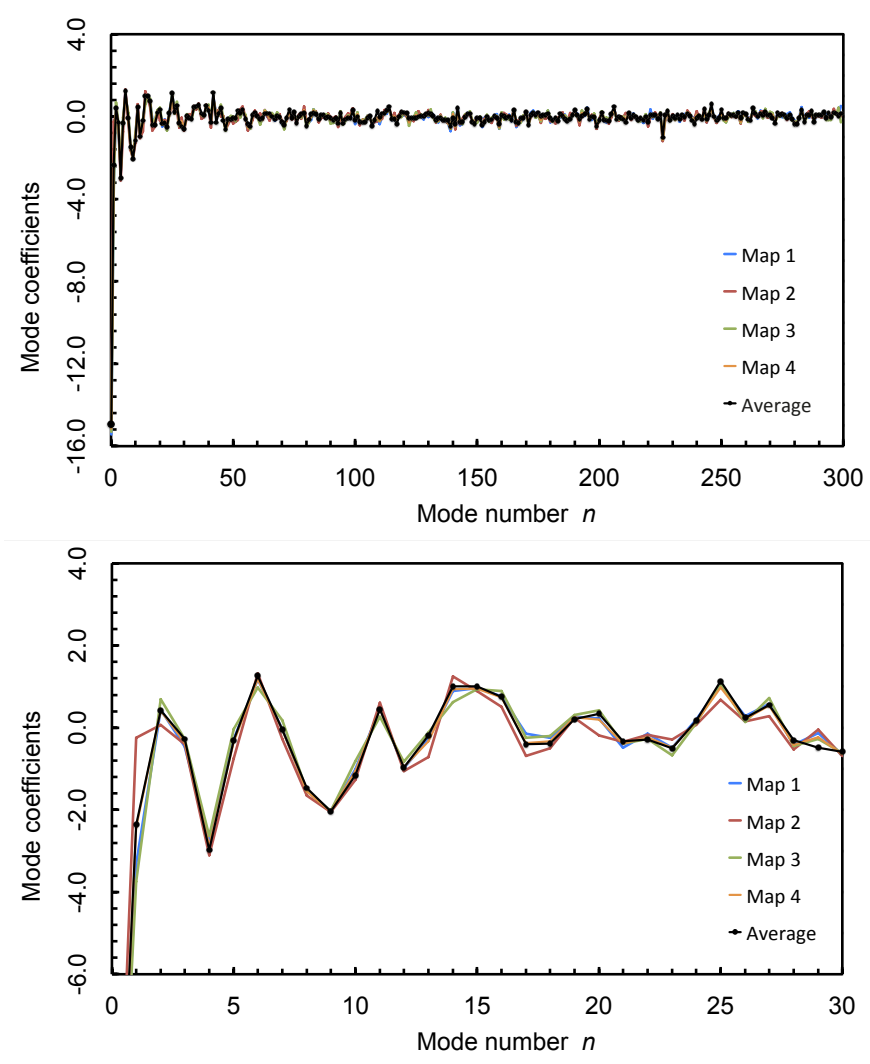

Fig. 11. Coefficients $\alpha_{n}^{R}$ Eq. (14) for the hybrid Fourier mode expansion of the cosmic string bispectrum Eq. (14). The average value $\alpha_{n}^{R}=\left\langle\beta_{n}^{R}\right\rangle$ (black line) is in remarkable agreement with all four string simulations as can be seen for $n<30$ (lower panel), with each exhibiting better than a $97 \%$ correlation overall.

$\beta_{n}^{R}$ for the four string simulations as shown in Fig. 11 for the Fourier modes. Agreement was good across all the $300 \alpha_{n}^{R}$ modes determined, as shown in detail for $n=1-30$ (see the lower panel of Fig. 11).

Quantitatively, the different string simulations produced bispectrum shapes that had above $97 \%$ correlations with each other (i.e., $\sum_{n} N^{-1} \alpha_{n}^{(1)} \alpha_{n}^{(2)}>0.97$ for $N^{2}=\sum_{n} \alpha_{n}^{(1) 2} \sum_{n} \alpha_{n}^{(2) 2}$ ). The overall integrated bispectrum amplitudes was consistent to within $4 \%$. Despite only four string map simulations, these are small errors relative to experimental and theoretical uncertainties. This robustness indicates that the overall string bispectrum signal at Planck resolution is a statistical summation of very many contributions from the millions of strings between the observer and the last scattering surface. To ensure the bispectrum $C_{l}$ weighting was not significantly affected by the presence of the large string signal, we repeated the modal extraction procedure for $G \mu / c^{2}=5 \times 10^{-7}$ (the string bispectrum amplitude reduced by a factor of 8 ). For the same string simulation, the shape correlations for different $G \mu / c^{2}$ were $99.4 \%$ or above and the amplitude scaled as expected with $\left(G \mu / c^{2}\right)^{3}$ to within $2 \%$. The string bispectrum shown in Fig. 11 is well converged with random errors from the averaging procedure small relative to the actual signal. We conclude that, assuming the physics and numerical accuracy of the string simulations that are available, we have extracted a string bispectrum of sufficient accuracy for the present non-Gaussian analysis.

The overall three-dimensional reconstruction of the string bispectrum shape is shown in Fig. 12, normalised in the usual way to approximately illustrate the signal-to-noise expected (that is, removing an overall $l^{-4}$ scaling, by dividing by the constant Sachs-Wolfe bispectrum shape). The first point to note is that the bispectrum is negative, reflecting the underlying string velocity correlations and curvature correlations that have created it; in the expanding universe, curved strings preferentially collapse, creating a negative temperature fluctuation towards the centre and a positive signal outside. In the overall spectrum, the $n=0$ mode is dominant, but it is modulated by other modes providing further interesting structure that could be described as broad arms extending along each axis (see Fig. 11); although somewhat "squeezed", the correlation with the local model is low. The string simulation power spectrum shown in Fig. 5 can be understood to be quantitatively modulating the string bispectrum away from the axes, with the signal slowly decaying beyond (say) $l_{1}, l_{2}>500$ in the $l_{3}$ direction.

The CMB bispectrum and trispectrum induced by the postrecombination gravitational effects of cosmic strings have been estimated analytically (Hindmarsh et al. 2009, 2010; Regan \& Shellard 2010). With simplifying assumptions, these predicted that the constant mode would be dominant with a broad central "equilateral" peak, but not the substructure observed in Fig. 12. In terms of missing physics in this post-recombination string bispectrum, we expect the recombination signal to lie in the range $\ell=200-1000$ (shown in the full NAMBU power spectrum in Fig. 3) and to significantly enhance the overall amplitude of the bispectrum (see also Landriau \& Shellard 2011, where recombination physics is included).

The correlation of the post-recombination string bispectrum with standard primordial shapes is small, because it does not contain an oscillatory component from the transfer functions. The local, equilateral and orthogonal bispectrum models correlate with strings at about $6 \%, 11 \%$ and $12 \%$, respectively. There is also a CMB ISW-lensing bispectrum $b_{\ell_{1} \ell_{2} \ell_{3}}^{\mathrm{ISW}}$ arising from the correlation between gravitational lensing and the late-time integrated Sachs-Wolfe effect arising from the underlying dominant Gaussian fluctuations (see, for example, the discussion in Planck Collaboration XXVI 2014). Like the string bispectrum, the ISW-lensing bispectrum is also mainly negative, but it is much more squeezed/flattened and correlates at only about $11 \%$ with the present string bispectrum. So the predicted ISW signal should provide a small positive bias for string detection of about $0.44 \sigma$. Diffuse point-source contamination is of greater concern because at $\ell_{\max }=2000$ this has a $40 \%$ anti-correlation with strings (for the simple Poisson-distributed point source template with $b_{\ell_{1} \ell_{2} \ell_{3}}^{\mathrm{PS}}=$ constant). This close relationship with point sources requires a joint analysis (see below). Other foreground contamination must also be considered, as we shall discuss, and for this we rely on realistic simulated FFP6 foreground residuals provided by the Planck component separation pipelines (see Planck Collaboration XII 2014).

\subsubsection{Planck string bispectrum results}

Using the modal bispectrum estimator, we have searched for the string bispectrum in the Planck CMB maps obtained using the foreground-separation techniques SMICA, NILC and SEVEM. We note that the modal estimator has passed through the full validation suite of NG tests described in the Planck Collaboration XXIV (2014), where further details about the analysis can be found. In summary, we have used the standard U73 mask, which includes a Galactic cut and a conservative point source mask, together with "inpainting" as in the $f_{\mathrm{NL}}$ analysis (essentially apodizing the mask). Together with the SMICA, NILC and SEVEM 


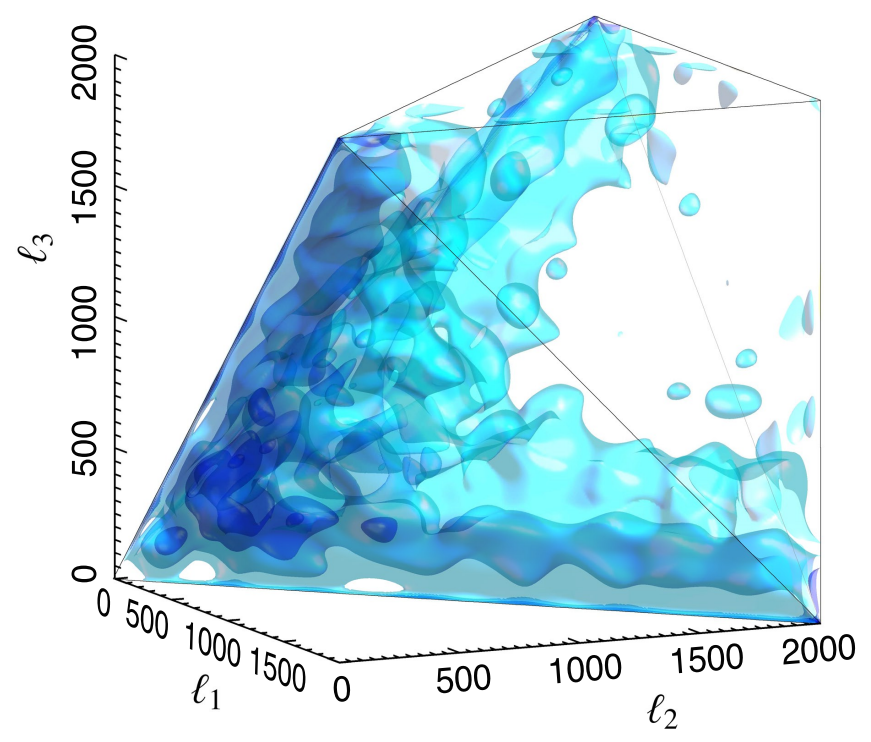

Fig. 12. Modal reconstruction of the post-recombination string bispectrum Eq. (14) extracted from Planck resolution map simulations. This is a $3 \mathrm{D}$ view of the allowed tetrahedral set of multipoles $\left(\ell_{1}, \ell_{2}, \ell_{3}\right)$ showing isosurfaces of the bispectrum density with darker blue for more negative values (it is normalised relative to the constant SW bispectrum).

foreground separated maps, realistic noise simulations as specified for the particular foreground-cleaned map, were used to determine the estimator's linear correction term and to determine the bispectrum variance, which was very nearly optimal. For calibration purposes, we always compare to the string model with tension $G \mu / c^{2}=1 \times 10^{-6}$ defining a string bispectrum parameter and normalising it to have $f_{\mathrm{NL}}=1$ in this case. The standard deviation $\Delta f_{\mathrm{NL}}=0.2$ obtained from this Planck analysis would imply a $5 \sigma$ detection of $G \mu / c^{2}=1 \times 10^{-6}$ strings. The strong scaling of the bispectrum amplitude on the string tension $\propto\left(G \mu / c^{2}\right)^{3}$, implies a given measurement yields $G \mu / c^{2}=\left(f_{\mathrm{NL}}\right)^{1 / 3} \times 10^{-6}$.

The results of the string bispectrum estimation for each of the SMICA, NILC and SEVEM maps are shown in Table 4. As shown by the non-Gaussian analysis (see Planck Collaboration XXIV 2014), the Planck data exhibits significant detections of both the predicted ISW-lensing bispectrum (above $2 \sigma$ ) and a residual point source signal (over $3 \sigma$ ). Since these may be confused with the string signal, we also quote their measurements in this Fourier mode basis. The ISW-lensing signal detection is described at length in Planck Collaboration XXVI 2014. The residual point source (PS) signal is discussed in Planck Collaboration XXIV 2014 where it is modelled as a simple Poissonian distribution. In principle, the PS amplitude can be extrapolated from source count models, but direct comparisons remain a goal for future Planck analysis. An independent analysis of the ISW-lensing, point source and string contributions to each map showed no evidence for a cosmic string signal (all estimates were were within $1 \sigma$, refer to the third column of Table 4). However, given the large contribution of diffuse point sources and their anti-correlation with the string bispectrum, we have also undertaken a joint analysis of the Planck data (which in this case is the same as marginalising over the point source signal). Before doing so, we subtract the expected ISW lensing signal that provides a $f_{\mathrm{NL}}=0.09$ string bias. The fifth column in Table 4 shows that marginalising point sources enhances the string signal up to the $2 \sigma$ level for all component separation methods; essentially the
Table 4. Modal bispectrum analysis of foreground-separated SMICA, NILC and SEVEM maps showing $f_{\mathrm{NL}}$ from strings, ISW-lensing and diffuse point sources.

\begin{tabular}{lllcc}
\hline \hline \multirow{5}{*}{ Method } & $\begin{array}{c}\text { Bispectrum } \\
\text { signal type }\end{array}$ & $\begin{array}{c}\text { Independent } \\
\text { analysis }\end{array}$ & $\begin{array}{c}\text { ISW subtract } \\
\text { joint }\end{array}$ & $\begin{array}{c}\text { ISW/FG res. } \\
\text { joint }\end{array}$ \\
\cline { 3 - 5 } SMICA & Lensing ISW & $0.75 \pm 0.37$ & - & - \\
& Diff. PS $\times 10^{28}$ & $1.05 \pm 0.32$ & $1.35 \pm 0.34$ & $1.40 \pm 0.34$ \\
& Cosmic strings & $0.19 \pm 0.20$ & $0.50 \pm 0.21$ & $0.37 \pm 0.21$ \\
& $G \mu / c^{2}(95 \%)$ & $8.4 \times 10^{-7}$ & $9.7 \times 10^{-7}$ & $9.3 \times 10^{-7}$ \\
NILC & Lensing ISW & $0.91 \pm 0.36$ & - & - \\
& Diff. PS $\times 10^{28}$ & $1.16 \pm 0.32$ & $1.44 \pm 0.34$ & $1.44 \pm 0.34$ \\
& Cosmic strings & $0.13 \pm 0.20$ & $0.46 \pm 0.21$ & $0.23 \pm 0.21$ \\
SEVEM & $G \mu / c^{2}(95 \%)$ & $8.1 \times 10^{-7}$ & $9.6 \times 10^{-7}$ & $8.7 \times 10^{-7}$ \\
& Lensing ISW & $0.6 \pm 0.36$ & - & - \\
& Diff. PS $\times 10^{28}$ & $1.07 \pm 0.35$ & $1.33 \pm 0.38$ & - \\
& Cosmic strings & $0.10 \pm 0.20$ & $0.38 \pm 0.21$ & - \\
& $G \mu / c^{2}(95 \%)$ & $7.9 \times 10^{-7}$ & $9.3 \times 10^{-7}$ & - \\
\hline
\end{tabular}

Notes. Three values for $f_{\mathrm{NL}}$ are given from independent analysis, joint point source/string analysis after ISW-lensing subtraction, and joint analysis after both ISW-lensing and foreground residual subtraction (FFP6).

constant mode becomes more strongly negative once the measured point sources are removed.

Other foregrounds, such as dust emission, could potentially produce spurious string signals if not subtracted properly. Foreground contamination has been extensively studied within the Planck simulation pipeline (FFP6) and foreground residual maps have been provided by each component separation team (including those for thermal dust, $\mathrm{CO}$ emission, free-free, synchrotron, spinning dust, kinetic and thermal SZ etc). We have analysed these residual FFP6 maps provided by both SMICA and NILC to seek evidence of correlations with the string bispectrum; individually these foregrounds make only small contributions (apart from PS), but cumulatively their effect cannot be neglected. The SMICA combined-residual map, without point sources and analysed with realistic noise, produces a string bias of $f_{\mathrm{NL}}=0.23$, which after ISW subtraction becomes $f_{\mathrm{NL}}=0.14$ (relative to a variance $\Delta f_{\mathrm{NL}}=0.20$ ). After both ISW and foreground residual subtraction, a joint analysis with point sources yields a SMICA string signal $f_{\mathrm{NL}}=0.37 \pm 0.21(\mathrm{Col} .5$ in Table 4). The apparent string bias in NILC from residual foregrounds was even higher $f_{\mathrm{NL}}=0.22$ (after ISW subtraction), meaning a joint analysis obtained $f_{\mathrm{NL}}=0.23 \pm 0.21$ (see Table 4).

We conclude, given our present understanding of point sources and foregrounds, that there does not appear to be significant evidence for a string bispectrum signal in the Planck nominal mission maps, so we infer the following post-recombination bispectrum constraint on strings:

$G \mu / c^{2}<9.0 \times 10^{-7} \quad(95 \%$ confidence $)$.

Here, we have taken the more conservative SMICA result $f_{\mathrm{NL}}=$ $0.37 \pm 0.21$, because of its preferred status for foreground separation techniques in Planck Collaboration XII (2014). The susceptibility of the string bispectrum to point source and other foreground contamination deserves further investigation and will require improved characterisation of the diffuse point source bispectrum (beyond the simple Poisson model), as well as 
identification of the specific foreground residuals which primarily contribute to the small string bias found in the FFP6 simulations.

The string bispectrum constraint Eq. (17) is a conservative upper limit on the string tension $G \mu / c^{2}$ because we have not included recombination contributions. Although this constraint is weaker than that from the power spectrum, it is an independent test for strings and the first quantitative string bispectrum limit to date. This should be considerably improved in future by inclusion of recombination physics and more precise foreground analysis. A comparison with the power spectrum amplitude indicates the string bispectrum should rise by $(2)^{3 / 2}$, which, together with the full mission data, would see the sensitivity improve by a factor of two (allowing constraints around $G \mu / c^{2}<4 \times 10^{-7}$ ). We note that the bispectrum is not the optimal non-Gaussian test for strings, because the string signal is somewhat suppressed by symmetry (the bispectrum cancels for straight strings). This fact motivates further study of the trispectrum, for which the Planck sensitivity is forecast to be $\Delta G \mu / c^{2} \approx 1 \times 10^{-7}$ (Fergusson et al. $2010 \mathrm{~b}$ ), as well as joint analysis of polyspectra.

\subsection{Steerable wavelet searches for cosmic strings}

Wavelets offer a powerful signal analysis tool due to their ability to localise signal content in scale (cf. frequency) and position simultaneously. Consequently, wavelets are well-suited for detecting potential CMB temperature contributions due to cosmic strings, which exhibit spatially localised signatures with distinct frequency content. Wavelets defined on the sphere are required to analyse full-sky Planck observations (see, for example, Freeden \& Windheuser 1997; Wiaux et al. 2005; Sanz et al. 2006; McEwen et al. 2006; Starck et al. 2006; Marinucci et al. 2008; Wiaux et al. 2008a).

We perform an analysis using the steerable wavelets on the sphere constructed by Wiaux et al. (2005). Here we exploit steerability to dynamically adapt the orientations analysed to the underlying data, performing frequentist hypothesis testing. We apply the first (1GD) and second (2GD) Gaussian derivative steerable wavelets, defined on the sphere through a stereographic projection, in order to search for cosmic strings in the Planck data. A steerable wavelet is a directional filter whose rotation by $\chi \in[0,2 \pi)$ about itself can be expressed in terms of a finite linear combination of non-rotated basis filters. Thus, the analysis of a signal with a given steerable wavelet $\Psi$ naturally identifies a set of wavelet coefficients, $W_{\Psi}\left(\omega_{0}, \chi, R\right)$, which describe the local features of the signal at each position $\omega_{0}$ on the sphere, for each orientation $\chi$ and for each physical scale $R$. Several local morphological properties can be defined in terms of the wavelet coefficients (Wiaux et al. 2008b), including the signed-intensity,

$I\left(\omega_{0}, R\right) \equiv W_{\Psi}\left(\omega_{0}, \chi_{0}, R\right)$

This quantity represents the value of the wavelet coefficient at the local orientation $\chi_{0}\left(\omega_{0}, R\right)$ that maximizes the absolute value of the wavelet coefficient itself. Let us emphasise that the signedintensity morphological property is a highly non-linear quantity, i.e., $I_{a+b}\left(\omega_{0}, R\right) \neq I_{a}\left(\omega_{0}, R\right)+I_{b}\left(\omega_{0}, R\right)$, where $a$ and $b$ are two signals on the sphere.

The presence of a cosmic string signal in the CMB is expected to leave a non-Gaussian signature that induces a modification in the distribution of $I\left(\omega_{0}, R\right)$ with respect to the lensed Gaussian case. We calibrated the dependence of these signatures on the string tension using four simulations of the cosmic string

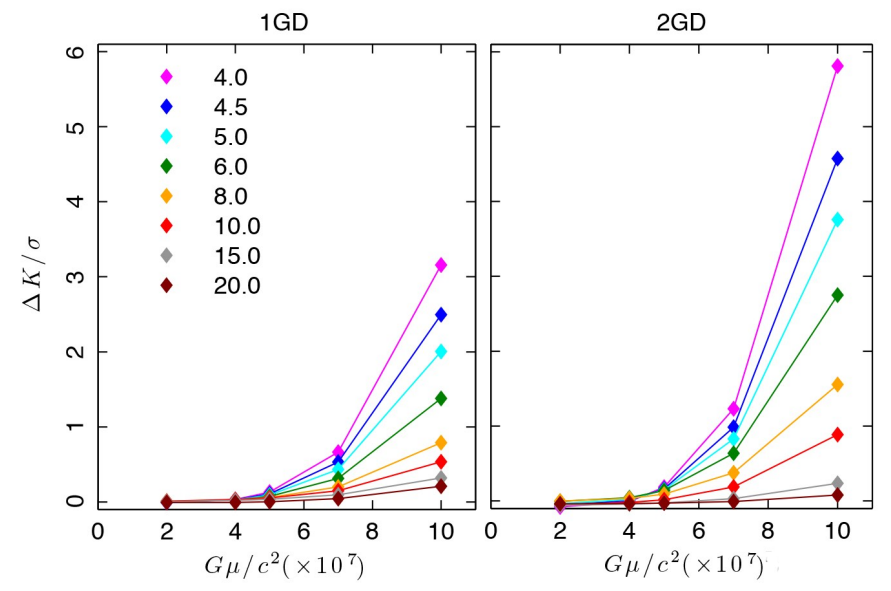

Fig. 13. Deviation of the kurtosis of the signed-intensity as a function of $G \mu / c^{2}$, normalised to the standard deviation of CMB and noise simulations: $\Delta K / \sigma=\left(K\left(R, G \mu / c^{2}\right)-K\left(R, G \mu / c^{2}=0\right)\right) / \sigma$. The left panel shows results for the 1GD wavelet and the right shows the $2 \mathrm{GD}$ wavelet. Each curve corresponds to a wavelet scale, $R$ (arcmin), included in the analysis. The final sensitivity of the method is determined by combining the two wavelets and all the scales.

contribution (Ringeval \& Bouchet 2012) combined with a set of lensed Gaussian CMB realisations, along with a realistic description of the Planck instrumental properties (refer to Planck Collaboration 2013).

A wide range of string tension values were explored, $G \mu / c^{2} \in\left[2.0 \times 10^{-7}, 1.0 \times 10^{-6}\right]$, considering several wavelet scales, $R=[4.0,4.5,5.0,6.0,8.0,10.0,15.0,20.0]$ arcmin. We use maps at a HEALPix resolution of $N_{\text {side }}=2048$, including multipoles up to $\ell_{\max }=2500$. We analyse the simulations with the same U73 mask of the Planck CMB map (refer to Planck Collaboration XII 2014), which masks both diffuse and compact foregrounds, leaving $73 \%$ of the sky remaining for further analysis (refer to discussion in Sect. 4.2.3). When computing the wavelet coefficients of a masked data set, artefacts are introduced close to the mask borders. We therefore define a set of exclusion masks such that, at each scale $R$, an extra region of the sky is excluded when computing any statistical measure. At each scale the exclusion mask is obtained by expanding the mask borders by three times the width of the corresponding wavelet.

The string non-Gaussian signatures are characterised in terms of the kurtosis of the signed-intensity $I\left(\omega_{0}, R\right)$ in Eq. (18) at the different scales $R$ and for both the 1GD and 2GD wavelets. The averaged results from the non-Gaussian simulations were used to model the distribution of the kurtosis as functions of $G \mu / c^{2}$, i.e., $K\left(R, G \mu / c^{2}\right)$. Other statistics, such as the skewness and the Higher-Criticism (e.g., Donoho \& Jin 2004; Cayón et al. 2005), have also been explored. We found that the kurtosis sensitivity to the string tension is higher than the alternative measures. In Fig. 13 we show the difference between the average kurtosis at several $G \mu / c^{2}$ values and the average kurtosis for $G \mu / c^{2}=0$, normalised to the standard deviation of the simulations. On the given range of scales, the 2GD wavelet appears to be more sensitive to the string signal. The final sensitivity of the method in recovering the string tension can be assessed from simulated data. We compare each simulation to the averaged model through a $\chi^{2}$ test, thus estimating $G \mu / c^{2}$ for the simulation. The test is performed jointly on the two wavelets for all the scales, and taking into account the correlations by means of a covariance matrix estimated from $\mathrm{CMB}$ and noise simulations. From the distribution 
of the $G \mu / c^{2}$ values recovered from the simulations the null hypothesis can be rejected at $95 \%$ CL for $G \mu / c^{2}>7 \times 10^{-7}$.

The same $\chi^{2}$ test was applied to the SEVEM CMB cleaned map, observing no evidence of a string signal at any of the scales studied. Despite the absence of strings, however, we noted that the $\chi^{2}$ of the best fit solution was higher than anticipated, corresponding to a probability to exceed (PTE) of $2 \%$. We identified the cause for such a poor fit to the null result as an incompatibility between the data and simulations at the smallest scales. We note that this incompatibility is not related to the potential presence of a string signal, since it biases the non-Gaussian estimators towards the opposite direction, as reflected also in the $\chi^{2}$ values. Similar behaviour is also noticed for the skewness of $I\left(\omega_{0}, R\right)$ and, to a lesser extent, for the Higher-Criticism measure.

We have extensively explored the causes of this incompatibility at small scales. First of all, we verified that such a behaviour is present also in the SMICA CMB solution, as well as SEVEM. We then analysed the half-ring half-difference SEVEM CMB maps, and we compared it to pure noise simulations. Indeed, half-ring maps are made from the data in the first half or second half of each stable pointing period, therefore we can obtain an estimate of the noise in the data by taking half of the difference between the two maps. We conclude that the half-ring noise estimate and noise simulations look compatible. We evaluated the impact on the estimator of unresolved point sources, both from radio galaxies and submillimetre star-forming galaxies, using simulations of these astrophysical components processed through the component separation pipelines (Planck Collaboration XII 2014). We found that at a wavelet scale of $R=4$ arcmin, these residual foregrounds induce a shift in the kurtosis, $\Delta K / \sigma=0.03$, i.e., a bias that is negligible for the present analysis. This shift only increases to more than $\Delta K / \sigma=0.3$ when extending the analysis to wavelet scales as small as $R=2.5$ arcmin. Finally, we also studied the SEVEM cleaned maps at 143 and $217 \mathrm{GHz}$, for which we considered wider galactic cuts (mask G56 and G35 from Planck Collaboration XV 2014). Since the inconsistency does not show a significant dependence on the frequency, there is no clear evidence for residual foreground contamination playing a major role.

At this point, it is worth stressing two important properties of the non-Gaussian statistics considered in this section. On the one hand, as mentioned above, it is a very non-linear estimator and, on the other, it depends on the local orientation of the sky signal. In fact, it is the only estimator considered in this paper that has such orientation-dependence. This is a critical point, since it implies that the simulations (lensed CMB, noise and foreground residuals) have to be more sophisticated if they are to capture the corresponding local anisotropic characteristics. This is especially difficult to achieve for the residual foregrounds, which are in part based on foreground models with small-scale fluctuations produced through isotropic and Gaussian extrapolations. As a consequence, we cannot discard completely either instrumental or foreground systematics at the smallest scales causing these incompatibilities. Nevertheless, we note that this lack of anisotropic modelling is not crucial for other estimators, as the angular power spectrum, or even the bispectrum, because these are isotropic quantities.

For the reasons given above, we have restricted our steerable wavelets analysis to scales $R \geq 6$ arcmin, where simulations are better understood for our specific purpose and where they appear compatible with the data. Analysing SEVEM on this angular range we find $G \mu / c^{2}<7 \times 10^{-7}$ at $95 \% \mathrm{CL}$. The next Planck release will continue to improve the modelling of instrumental and foreground properties and, therefore, we expect to be able to fully exploit the steerable wavelet capabilities down to the smallest scales $(R=2.5 \mathrm{arcmin})$. Based on simulations, this can significantly improve the sensitivity of the estimator, allowing constraints of $G \mu / c^{2}<4 \times 10^{-7}$ at $95 \%$ CL.

Finally, we note that we have also endeavoured to study the simulated string maps using spherical wavelets, making an extension of previous work (Wiaux et al. 2008a; Hammond et al. 2009) to compute the Bayesian posterior distribution of the string tension. Both the spherical and steerable wavelet methods offer good prospects for improved non-Gaussian string constraints from the Planck full mission data.

\subsection{Real space tests for cosmic strings}

\subsubsection{Minkowski functionals method}

Minkowski Functionals (MFs) describe morphological properties of the CMB field, and can be used as generic estimators of non-Gaussianities (Planck Collaboration XXIII 2014). However, they have been considered to constrain specific NG models such as cosmic strings, for example on gradient temperature maps (see e.g., Gott et al. 1990). Indeed they have sensitivity to nonGaussianity sourced by strings at all orders (i.e., including the kurtosis or trispectrum) and they could prove to be a powerful tool to constrain topological defects in general. For the sake of brevity and conciseness, precise definitions of MFs and analytic formulations are presented in Planck Collaboration XXIII (2014) and, here, we only review how MFs can be used to constrain the string energy density $G \mu / c^{2}$. We follow the Bayesian method discussed in Ducout et al. (2012) for the local model $f_{\mathrm{NL}}^{\text {local }}$ but which can quite similarly be applied to the string case.

We measure the four normalised ${ }^{2}$ functionals $v_{k}(k=0,3)$ (respectively Area, Perimeter, Genus and $N_{\text {cluster }}$ ), computed on $n_{\text {th }}=26$ thresholds $v$, between $v_{\text {min }}=-3.5$ and $v_{\max }=+3.5$ in units of the standard deviation of the map. The four functionals are similarly sensitive to cosmic strings and we build a statistics using the combination of all functionals, forming one vector $y$ (of size $n=104$ ).

The principle of this Bayesian method is to compare measurement of MFs on Planck data $\hat{y}$ to the MFs model curve for strings at the level $G \mu / c^{2}, y\left(G \mu / c^{2}\right)$. The Bayes formula is

$$
P\left(G \mu / c^{2} \mid \hat{y}\right)=\frac{P\left(\hat{y} \mid G \mu / c^{2}\right) P\left(G \mu / c^{2}\right)}{\int P\left(\hat{y} \mid G \mu / c^{2}\right) P\left(G \mu / c^{2}\right) \mathrm{d} G \mu / c^{2}} .
$$

We take a flat prior for the parameter $G \mu / c^{2}$, with $P\left(G \mu / c^{2}\right)$ being a constant over a reasonable range of values for $G \mu / c^{2}$, mostly determined from previous experiments (between 0 and 1e-6). The evidence $\int P\left(\hat{y} \mid G \mu / c^{2}\right) P\left(G \mu / c^{2}\right) \mathrm{d} G \mu / c^{2}$ is being considered as a normalisation factor.

The likelihood $P\left(\hat{y} \mid G \mu / c^{2}\right)$ is a multivariate Gaussian: $v_{\max }$ was chosen not too extreme, to avoid sensitivity to rare events, and the map's filtering allows to probe a sufficient number of independent harmonic modes to keep fluctuations small. The likelihood's Gaussianity has also been verified systematically at each point, using simulations. More details on the likelihood are

\footnotetext{
2 Raw Minkowski functionals $V_{k}$ depend on the Gaussian part of fields through a normalisation factor $A_{k}$, that is a function only of the power spectrum shape. We therefore normalise functionals $v_{k}=V_{k} / A_{k}$ to focus on non-Gaussianity, see Planck Collaboration XXIII (2014) and references therein.
} 
discussed in Gott et al. (1990); Ducout et al. (2012). This allows us to define a simple $\chi^{2}$ test for $G \mu / c^{2}$ and the posterior becomes

$P\left(G \mu / c^{2} \mid \hat{y}\right) \propto \exp \left[-\frac{\chi^{2}\left(\hat{y}, G \mu / c^{2}\right)}{2}\right]$,

with

$\chi^{2}\left(\hat{y}, G \mu / c^{2}\right) \equiv\left[\hat{y}-y\left(G \mu / c^{2}\right)\right]^{T} C^{-1}\left[\hat{y}-y\left(G \mu / c^{2}\right)\right]$.

The covariance matrix $C$ is computed from $10^{4}$ Gaussian simulations $^{3}$ : to verify the Gaussian approximation, tests have been performed using non-Gaussian matrices over a dozen values of $G \mu / c^{2}$ and covariance matrices and $\chi^{2}$ remained constant within numerical precision; furthermore the number of Gaussian simulations used to estimate the covariance matrix guarantees the stability of the statistics for these threshold values (see convergence tests in Ducout et al. 2012).

The cosmic string model curve $y\left(G \mu / c^{2}\right) \equiv \bar{y}\left(G \mu / c^{2}\right)$ is calibrated on $10^{3}$ realistic lensed Planck simulations, to which we have added a string component at a specified level. These simulations take into account the asymmetry of beams and the component separation process (FFP6 simulations, see Planck Collaboration 2013, for a detailed description). For the string component, we had at our disposal only two high resolution string map simulations (Ringeval \& Bouchet 2012), so our model is the averaged curve obtained from this combination of Planck and string simulations.

Due to the non-linear dependence of MFs on $G \mu / c^{2}$ and the small number of string simulations, the posterior distribution is quite complex and noisy. For this reason, we evaluated the posterior at $n_{\mathrm{NL}}=51$ values of $G \mu / c^{2}$, between 0 and $10 \times 10^{-7}$, to obtain our Planck estimate for $G \mu / c^{2}$. This estimate is stable and has been validated in realistic conditions with the Planck String Challenges described above, and for which we found consistent results with the underlying (unknown) $G \mu / c^{2}$.

\subsubsection{Minkowski functionals results}

For the constraint on $G \mu / c^{2}$, we analysed the foregrounds separated SMICA map at $N_{\text {side }}=2048$ and $\ell_{\text {max }}=2000$, using the U73 mask ( $f_{\text {sky }}=73 \%$ of the sky is unmasked). The smallest point sources holes were inpainted. We applied two specific Wiener filters to the map, designed to enhance the information from the map itself $\left(W_{\mathrm{M}}\right)$ and from the gradients of the map $\left(W_{\mathrm{D} 1}=\sqrt{\ell(\ell+1)} W_{\mathrm{M}}\right)$. The filters are shown in Fig. 14 .

Additionally, we estimated the average impact of some residual foregrounds and secondaries (FG) on $G \mu / c^{2}$, using the linear properties of MFs and foregrounds models processed through the Planck simulation pipeline (FFP6 simulations). Uncorrelated (Poissonian) unresolved point sources (PS), Cosmic Infrared Background (CIB) and Sunyaev-Zeldovich cluster ${ }^{4}$ (SZ) signals can be introduced as a simple additive bias $\Delta \bar{y}^{\mathrm{PS}, \ldots}$ on MFs' curves following:

$\hat{y}=\hat{y}^{\mathrm{FGsubtracted}}+\Delta \bar{y}^{\mathrm{PS}}+\Delta \bar{y}^{\mathrm{CIB}}+\Delta \bar{y}^{\mathrm{SZ}}$.

These biases are obtained as the average obtained on 100 simulations.

\footnotetext{
3 The Gaussian simulations endeavour to incorporate realistic noise from the Planck data, but only the effective isotropic beam of the component separation method.

4 The SZ signal does not include the $\mathrm{SZ} \times$ lensing NG contribution.
}

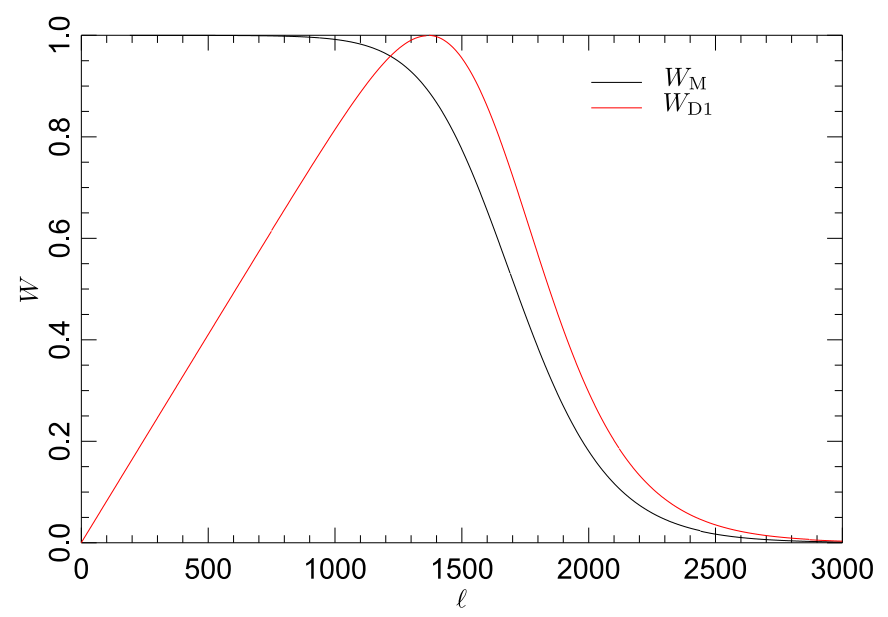

Fig. 14. The two Wiener filters, $W_{\mathrm{M}}$ and $W_{\mathrm{D} 1}$, used to constrain $G \mu / c^{2}$ with Minkowski functionals.

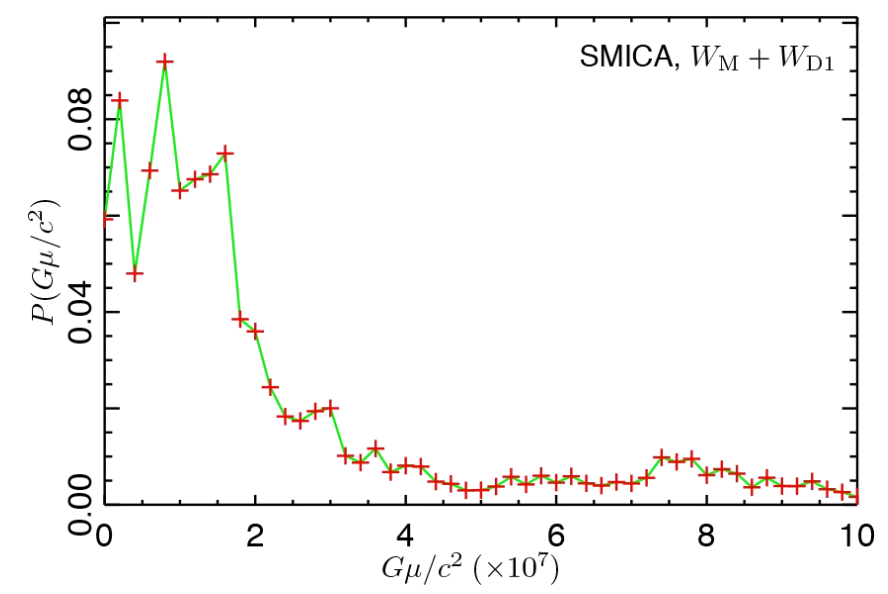

Fig. 15. Posterior distribution of the parameter $G \mu / c^{2}$ obtained with Minkowski functionals. This estimate takes into account the lensing of the data, but not the effects of foreground residuals.

We eventually obtain the posterior distribution of $G \mu / c^{2}$ (Fig. 15). As mentioned previously, the posterior curve is complex and noisy and so as to give an estimate of $G \mu / c^{2}$ we report its credible interval at $95 \%{ }^{5}$. Results are summarized in Table 5, for raw data (lensing only is accounted for and subtracted) and foreground subtracted data (lensing, PS, CIB and SZ are considered). The discrepancy between the two filters can be explained because the derivative filter $W_{\mathrm{D} 1}$ scans smaller scales than $W_{\mathrm{M}}$ so it is more easily biased by foreground residuals. Given the remaining foreground uncertainties, we take the most conservative of the MFs constraints for the cosmic string contribution to the Planck data to be

$G \mu / c^{2}<7.8 \times 10^{-7} \quad$ at $95 \%$ C.I.

The corresponding posterior is presented in Fig. 15.

Some caveats need to be mentioned that may influence these results. First, for the MF method itself, an important limitation is the small number of string simulations used to calibrate the estimator. The estimator appears to be mostly sensitive to lowredshift strings (infinite strings, with redshifts between 0 and 30 ), and this is affected by cosmic variance. As low-redshift string simulations are much faster to produce than complete

5 This is defined as $95 \%$ of the $G \mu / c^{2}$ posterior curve integral being contained between 0 and the estimate. 
Table 5. MFs constraints obtained on $G \mu / c^{2}$, at the $95 \%$ C.I.

\begin{tabular}{ccc}
\hline \hline \multirow{2}{*}{ Map } & \multicolumn{2}{c}{$G \mu / c^{2}$} \\
\cline { 2 - 3 } & $W_{\mathrm{M}}$ & $W_{\mathrm{M}}+W_{\text {D1 }}$ \\
\hline Raw $\ldots \ldots \ldots \ldots$ & $<6.8 \times 10^{-7}$ & $<7.8 \times 10^{-7}$ \\
FG subtracted $\ldots \ldots$ & $<6.0 \times 10^{-7}$ & $<3.6 \times 10^{-7}$ \\
\hline
\end{tabular}

Notes. These results are obtained on the SMICA map with the U73 mask $\left(f_{\text {sky }}=0.73\right)$. The "Raw map" result includes only the lensing contribution to the data, while the "Foreground subtracted map" includes the lensing, Poissonian point sources, CIB and SZ clusters contributions.

simulations back to recombination, it should be possible to improve the robustness of the constraint using these relatively soon. Secondly, the impact of Galactic residuals should be assessed in further detail, especially for the filter $W_{\mathrm{D} 1}$ that we have observed to be less robust against residuals than the $W_{\mathrm{M}}$ filter.

With advances in studying these experimental effects there are good prospects for the full mission data, the sensitivity of the MFs estimator should improve substantially, with simulations forecasting possible MF cosmic string constraints of $G \mu / c^{2}<3 \times 10^{-7}$ at the $95 \%$ C.I.

We note that further real space analysis of string map simulations has been undertaken with scaling indices of the pixel temperature distribution (see, e.g., Räth et al. 2011). Extensions calculating a set of anisotropic scaling indices along predefined directions appear to offer good prospects for string detection.

\section{Conclusions}

We have reviewed the signatures induced by cosmic strings in the CMB and searched for these in the Planck data, resulting in new more stringent constraints on the dimensionless string tension parameter $G \mu / c^{2}$. A pre-requisite for accurate constraints on cosmic strings is a quantitative understanding of both cosmological string network evolution and the effects they induce in the CMB. These are computationally demanding problems but progress has been made recently on several fronts: first, high resolution simulations of Nambu-Goto strings have yielded robust results for the scale-invariant properties of string networks on large scales, while there has been increasing convergence about small-scale structure and loops (for which the CMB predictions are less sensitive). Secondly, post-recombination gravitational effects of strings have been incorporated into full-sky Planck resolution $\mathrm{CMB}$ temperature maps that are important for validating non-Gaussian search methods. Finally, fast Boltzmann pipelines to calculate $\mathrm{CMB}$ power spectra induced by causal sources have been developed and tested at high resolution, whether for field theory simulations of strings or textures or for models of Nambu-Goto strings. Three-dimensional field theory simulations of vortex-strings at sufficient resolution should, in principle, converge towards the one-dimensional Nambu-Goto string simulations, but testing this is not numerically feasible at present. For this reason, we believe it is prudent to also include constraints on field theory strings (labelled $G \mu_{\mathrm{AH}}$ ), thus encompassing cosmic string models for which radiative effects are important at late times (such as global strings). We believe this brackets the important theoretical uncertainties that remain, that is, we have used the best available information to constrain both Nambu-Goto strings (NAMBU) and field theory strings $(\mathrm{AH})$. This work has also obtained more stringent constraints on semilocal strings and global textures.

\subsection{Cosmic string constraints and the CMB power spectrum}

Accurate forecasts for the CMB power spectrum induced by cosmic strings are more difficult to compute than their equivalent for simple adiabatic inflationary scenarios. It requires knowledge of the source, quantified by the unequal-time correlator (UETC) of the defect stress-energy tensor, from well before recombination to the present day, which is not computationally feasible. Fortunately, we can exploit scale-invariant string evolution to extrapolate the results of simulations with substantially smaller dynamic range. We use two methods to obtain predictions for the UETCs. An unconnected segment model (USM) is used to model the properties of an evolving string network, determining its density from an analytic one-scale evolution model, and the sources are coupled to the line-of-sight Boltzmann solver CMBACT. A second independent pipeline measures the UETCs directly from string simulations in the Abelian-Higgs field theory, passing these to a modified form of the CMBEASY Boltzmann code. The resulting Nambu-Goto and Abelian-Higgs string CMB power spectra are illustrated in Fig. 3. Free parameters in the USM model can be chosen to phenomenologically match the field theory UETCs (denoted the AH-mimic model) and the comparison is also shown in Fig. 3, validating the two independent pipelines.

To compute constraints on cosmic string scenarios we have added the angular power spectrum to that for a simple adiabatic model, assuming that they are uncorrelated, with the fraction of the spectrum contributed by cosmic strings being $f_{10}$ at $\ell=10$. This has been added to the standard 6 parameter fit using COSMOMC with flat priors. For the USM models we have obtained the constraint for the Nambu-Goto string model

$G \mu / c^{2}<1.5 \times 10^{-7}, \quad f_{10}<0.015$,

while for the Abelian-Higgs field theory model we find,

$G \mu_{\mathrm{AH}} / c^{2}<3.2 \times 10^{-7}, \quad f_{10}<0.028$.

The marginalised likelihoods for $f_{10}$ and in the $f_{10}-\Omega_{\mathrm{b}} h^{2}$ plane were presented in Fig. 10. With Planck nominal mission data these limits are already about a factor of two more stringent than the comparable WMAP 7-year string constraints and these Planck limits improve further with the inclusion of high- $\ell$ data.

\subsection{Non-Gaussian searches for cosmic strings}

Complementary searches for non-Gaussian signatures from cosmic strings were performed and we have reported constraints from the string bispectrum, steerable wavelets and Minkowski functionals. These methods participated in the Planck String Challenges and have undergone non-Gaussian validation tests.

The post-recombination string bispectrum has been reconstructed and calibrated from string-induced CMB maps using a modal estimator. String challenge analysis with Planck-realistic noise simulations and mask indicated a nominal mission sensitivity of $\Delta G \mu / c^{2} \approx 5.8 \times 10^{-7}$. Analysis of SMICA, NILC and SEVEM foreground-separated maps has yielded $f_{\mathrm{NL}}=0.37 \pm 0.21$ for the string bispectrum shape, which translates into a bispectrum constraint on the string tension, $G \mu / c^{2}<9.0 \times 10^{-7}$ (95\% CL). Steerable wavelet methods have been calibrated on string simulation maps added to Gaussian CMB maps with realistic noise and masking, showing a sensitivity of up to $\Delta G \mu / c^{2} \approx 4 \times 10^{-7}$. The string signal was shown to have greater impact on the kurtosis of the signed-intensity than on its skewness, and no evidence of a string signal was found in the Planck 
data. Minkowski functionals have been applied to string simulation maps in a Planck-realistic context, computing the four functionals - area, perimeter, genus and $N_{\text {cluster }}-$ after application of Weiner filters. Using these distributions, a Bayesian estimator has been constructed to constrain the string tension. Analysis of the SMICA foreground-cleaned maps yielded a MF constraint of $G \mu / c^{2}<7.8 \times 10^{-7}(95 \% \mathrm{CL})$.

Non-Gaussian searches for strings are complementary to the power spectrum analysis and yield constraints as low as $G \mu / c^{2}<$ $7.8 \times 10^{-7}$, though we note the potential impact of foreground residuals in limiting current precision. These are conservative upper bounds because they only include post-recombination string contributions, unlike the string power spectrum analysis. Having such a broad suite of tools, ranging from multipole space, through wavelets, to real space detection methods, allows cross-validation and reinforces the conclusion that there is at present no evidence for cosmic strings in the Planck nominal mission data.

Acknowledgements. The development of Planck has been supported by: ESA; CNES and CNRS/INSU-IN2P3-INP (France); ASI, CNR, and INAF (Italy); NASA and DoE (USA); STFC and UKSA (UK); CSIC, MICINN, JA and RES (Spain); Tekes, AoF and CSC (Finland); DLR and MPG (Germany); CSA (Canada); DTU Space (Denmark); SER/SSO (Switzerland); RCN (Norway); SFI (Ireland); FCT/MCTES (Portugal); and PRACE (EU). A description of the Planck Collaboration and a list of its members, including the technical or scientific activities in which they have been involved, can be found at http://www.sciops.esa.int/index.php? project $=$ planck\&page $=\mathrm{Pl}$ lanck_Collaboration. We also wish to acknowledge the use of the COSMOS supercomputer, part of the DiRAC HPC Facility funded by STFC and the UK Large Facilities Capital Fund, use of the Andromeda cluster of the University of Geneva, and resources of the National Energy Research Scientific Computing Center.

\section{References}

Achucarro, A., Celi, A., Esole, M., Van den Bergh, J., \& Van Proeyen, A. 2006, J. High Energy Phys., 0601, 102

Albrecht, A., \& Turok, N. 1989, Phys. Rev. D, 40, 973

Albrecht, A., Battye, R. A., \& Robinson, J. 1997, Phys. Rev. Lett., 79, 4736

Albrecht, A., Battye, R. A., \& Robinson, J. 1999, Phys. Rev. D, 59, 3508

Allen, B., \& Shellard, P. 1990, Phys. Rev. Lett., 64, 119

Allen, B., Caldwell, R., Shellard, E., Stebbins, A., \& Veeraraghavan, S. 1996, Phys. Rev. Lett., 77, 3061

Avgoustidis, A., Copeland, E. J., Moss, A., \& Skliros, D. 2012, Phys. Rev. D, 86,3513

Battye, R., \& Moss, A. 2010, Phys. Rev. D, 82, 023521

Battye, R. A., Garbrecht, B., \& Moss, A. 2006, JCAP, 0609, 007

Battye, R. A., Garbrecht, B., Moss, A., \& Stoica, H. 2008, JCAP, 0801, 020

Battye, R., Garbrecht, B., \& Moss, A. 2010, Phys. Rev. D, 81, 3512

Bennett, D. P., \& Bouchet, F. R. 1989, Phys. Rev. Lett., 63, 2776

Bennett, D. P., \& Bouchet, F. R. 1990, Phys. Rev. D, 41, 2408

Bevis, N., Hindmarsh, M., \& Kunz, M. 2004, Phys. Rev. D, 70, 3508

Bevis, N., Hindmarsh, M., Kunz, M., \& Urrestilla, J. 2007a, Phys. Rev. D, 76, 3005

Bevis, N., Hindmarsh, M., Kunz, M., \& Urrestilla, J. 2007b, Phys. Rev. D, 75, 5015

Bevis, N., Hindmarsh, M., Kunz, M., \& Urrestilla, J. 2008, Phys. Rev. Lett., 100, 1301

Bevis, N., Hindmarsh, M., Kunz, M., \& Urrestilla, J. 2010, Phys. Rev. D, 82, 5004

Blanco-Pillado, J. J., Olum, K. D., \& Shlaer, B. 2011, Phys. Rev. D, 83, 3514

Bouchet, F. R., Bennett, D. P., \& Stebbins, A. 1988, Nature, 335, 410

Cayón, L., Jin, J., \& Treaster, A. 2005, MNRAS, 362, 826

Copeland, E. J., \& Kibble, T. 2010, Proc. Roy. Soc. London A, 466, 623

Cruz, M., Turok, N., Vielva, P., Martinez-Gonzalez, E., \& Hobson, M. 2007, Science, 318, 1612

Dasgupta, K., Hsu, J. P., Kallosh, R., Linde, A. D., \& Zagermann, M. 2004, J. High Energy Phys., 0408, 030

Dasgupta, K., Firouzjahi, H., \& Gwyn, R. 2007, J. High Energy Phys., 0704, 093

Donoho, D., \& Jin, J. 2004, Ann. Stat., 32, 962

Doran, M. 2005, JCAP, 0510, 011
Ducout, A., Bouchet, F. R., Colombi, S., \& Pogosyan, D. 2013, MNRAS, 429, 2104

Dunkley, J., Hlozek, R., Sievers, J., et al. 2011, ApJ, 739, 52

Durrer, R., Kunz, M., \& Melchiorri, A. 2002, Phys. Rept., 364, 1

Dvorkin, C., Wyman, M., \& Hu, W. 2011, Phys. Rev. D, 84, 3519

Feeney, S. M., Johnson, M. C., Mortlock, D. J., \& Peiris, H. V. 2012, Phys. Rev. Lett., 108, 241301

Feeney, S. M., Johnson, M. C., McEwen, J. D., Mortlock, D. J., \& Peiris, H. V. 2013, Phys. Rev. D, 88, 043012

Fergusson, J., \& Shellard, E. 2009, Phys. Rev. D, 80, 3510

Fergusson, J., Liguori, M., \& Shellard, E. 2010a, Phys. Rev. D, 82, 3502

Fergusson, J., Regan, D., \& Shellard, E. 2010b, unpublished [arXiv: 1012.6039]

Foreman, S., Moss, A., \& Scott, D. 2011, Phys. Rev. D, 84, 3522

Fraisse, A. A., Ringeval, C., Spergel, D. N., \& Bouchet, F. R. 2008, Phys. Rev. D, 78, 3535

Freeden, W., \& Windheuser, U. 1997, Appl. Comput. Harmon. Anal., 4, 1

Gott III, J. R. 1985, ApJ, 288, 422

Gott, J. R. I., Park, C., Juszkiewicz, R., et al. 1990, ApJ, 352, 1

Hammond, D. K., Wiaux, Y., \& Vandergheynst, P. 2009, MNRAS, 398, 1317

Hindmarsh, M. 1994, ApJ, 431, 534

Hindmarsh, M. 2011, Prog. Theor. Phys. Suppl., 190, 197

Hindmarsh, M., \& Kibble, T. 1995, Rept. Prog. Phys., 58, 477

Hindmarsh, M., Ringeval, C., \& Suyama, T. 2009, Phys. Rev. D, 80, 083501

Hindmarsh, M., Ringeval, C., \& Suyama, T. 2010, Phys. Rev. D, 81, 063505

Hou, Z., Reichardt, C. L., Story, K. T., et al. 2014, ApJ, 782, 74

Jeannerot, R., Rocher, J., \& Sakellariadou, M. 2003, Phys. Rev. D, 68, 103514

Kaiser, N., \& Stebbins, A. 1984, Nature, 310, 391

Kibble, T. 1976, J. Phys., A9, 1387

Kibble, T. 1985, Nucl. Phys. B, 252, 227

Landriau, M., \& Shellard, E. 2003, Phys. Rev. D, 67, 103512

Landriau, M., \& Shellard, E. 2011, Phys. Rev. D, 83, 043516

Lizarraga, J., Sendra, I., \& Urrestilla, J. 2012, Phys.Rev. D, 86, 123014

Marinucci, D., Pietrobon, D., Balbi, A., et al. 2008, MNAS, 383, 539

Martins, C., \& Shellard, E. 1996, Phys. Rev. D, 54, 2535

Martins, C., \& Shellard, E. 2006, Phys. Rev. D, 73, 043515

McEwen, J. D., Hobson, M. P., \& Lasenby, A. N. 2006, unpublished [arXiv: astro-ph/0609159]

Moore, J. N., Shellard, E. P. S., \& Martins, C. J. A. P. 2001, Phys. Rev. D 65, 3503

Moore, J., Shellard, E., \& Martins, C. 2002, Phys. Rev. D, 65, 023503

Pen, U.-L., Seljak, U., \& Turok, N. 1997, Phys. Rev. Lett., 79, 1611

Planck Collaboration 2013, The Explanatory Supplement to the Planck 2013 results, http://www.sciops.esa.int/wikiSI/planckpla/index.php? title=Main_Page (ESA)

Planck Collaboration I. 2014, A\&A, 571, A1

Planck Collaboration II. 2014, A\&A, 571, A2

Planck Collaboration III. 2014, A\&A, 571, A3

Planck Collaboration IV. 2014, A\&A, 571, A4

Planck Collaboration V. 2014, A\&A, 571, A5

Planck Collaboration VI. 2014, A\&A, 571, A6

Planck Collaboration VII. 2014, A\&A, 571, A7

Planck Collaboration VIII. 2014, A\&A, 571, A8

Planck Collaboration IX. 2014, A\&A, 571, A9

Planck Collaboration X. 2014, A\&A, 571, A10

Planck Collaboration XI. 2014, A\&A, 571, A11

Planck Collaboration XII. 2014, A\&A, 571, A12

Planck Collaboration XIII. 2014, A\&A, 571, A13

Planck Collaboration XIV. 2014, A\&A, 571, A14

Planck Collaboration XV. 2014, A\&A, 571, A15

Planck Collaboration XVI. 2014, A\&A, 571, A16

Planck Collaboration XVII. 2014, A\&A, 571, A17

Planck Collaboration XVIII. 2014, A\&A, 571, A18

Planck Collaboration XIX. 2014, A\&A, 571, A19

Planck Collaboration XX. 2014, A\&A, 571, A20

Planck Collaboration XXI. 2014, A\&A, 571, A21

Planck Collaboration XXII. 2014, A\&A, 571, A22

Planck Collaboration XXIII. 2014, A\&A, 571, A23

Planck Collaboration XXIV. 2014, A\&A, 571, A24

Planck Collaboration XXV.2014, A\&A, 571, A25

Planck Collaboration XXVI. 2014, A\&A, 571, A26

Planck Collaboration XXVII. 2014, A\&A, 571, A27

Planck Collaboration XXVIII. 2014, A\&A, 571, A28

Planck Collaboration XXIX. 2014, A\&A, 571, A29

Planck Collaboration XXX. 2014, A\&A, 571, A30

Planck Collaboration XXXI. 2014, A\&A, 571, A31

Pogosian, L., \& Vachaspati, T. 1999, Phys. Rev. D, 60, 083504

Polchinski, J. 2005, Int. J. Mod. Phys., 20, 3413 
Räth, C., Banday, A. J., Rossmanith, G., et al. 2011, MNRAS, 415, 2205 Regan, D. M., \& Shellard, E. P. S. 2010, Phys. Rev. D, 82, 3527 Ringeval, C. 2010, Adv. Astron., 2010, 0507

Ringeval, C., \& Bouchet, F. R. 2012, Phys. Rev. D, 86, 3513

Ringeval, C., Sakellariadou, M., \& Bouchet, F. 2007, JCAP, 0702, 023

Sanidas, S., Battye, R., \& Stappers, B. 2012, Phys. Rev. D, 85, 122003

Sanz, J. L., Herranz, D., López-Caniego, M., \& Argüeso, F. 2006, in Proc. 14th Europ. Signal Processing Conf. EUSIPCO

Sievers, J. L., Hlozek, R. A., Nolta, M. R., et al. 2013, JCAP, 10, 060

Starck, J.-L., Moudden, Y., Abrial, P., \& Nguyen, M. 2006, A\&A, 446, 1191

Stebbins, A. 1988, ApJ, 327, 584

Stebbins, A., \& Veeraraghavan, S. 1995, Phys. Rev. D, 51, 1465

Takahashi, K., Naruko, S., Matsumoto, R., et al. 2009, JCAP, 0910, 003

Urrestilla, J., Achucarro, A., \& Davis, A. 2004, Phys. Rev. Lett., 92, 1302

Urrestilla, J., Bevis, N., Hindmarsh, M., Kunz, M., \& Liddle, A. R. 2008, JCAP, 0807, 010

Urrestilla, J., Bevis, N., Hindmarsh, M., \& Kunz, M. 2011, JCAP, 1112, 021

Vachaspati, T., \& Achucarro, A. 1991, Phys. Rev. D, 44, 3067

van Haasteren, R., Levin, Y., Janssen, G., et al. 2011, MNRAS, 414, 3117

Vilenkin, A. 1981a, Phys. Rev. Lett., 46, 1169

Vilenkin, A. 1981b, Phys. Lett. B, 107, 47

Vilenkin, A., \& Shellard, E. 2000, Cosmic Strings and Other Topological Defects, Cambridge Monographs on Mathematical Physics (Cambridge University Press)

Vincent, G., Antunes, N. D., \& Hindmarsh, M. 1998, Phys. Rev. Lett., 80, 2277

Wiaux, Y., Jacques, L., \& Vandergheynst, P. 2005, ApJ, 632, 15

Wiaux, Y., McEwen, J. D., Vandergheynst, P., \& Blanc, O. 2008a, MNRAS, 388, 770

Wiaux, Y., Vielva, P., Barreiro, R. B., Martinez-González, E., \& Vandergheynst, P. 2008b, MNRAS, 385, 939

Yamauchi, D., Sendouda, Y., Yoo, C.-M., et al. 2010a, JCAP, 1005, 033

Yamauchi, D., Takahashi, K., Sendouda, Y., Yoo, C.-M., \& Sasaki, M. 2010b, Phys. Rev. D, 82, 063518

Zeldovich, Y. 1980, MNRAS, 192, 663

1 APC, AstroParticule et Cosmologie, Université Paris Diderot, CNRS/IN2P3, CEA/lrfu, Observatoire de Paris, Sorbonne Paris Cité, 10 rue Alice Domon et Léonie Duquet, 75205 Paris Cedex 13, France

2 Aalto University Metsähovi Radio Observatory, Metsähovintie 114, 02540 Kylmälä, Finland

3 African Institute for Mathematical Sciences, 6-8 Melrose Road, Muizenberg, 7701 Rondebosch Cape Town, South Africa

4 Agenzia Spaziale Italiana Science Data Center, c/o ESRIN, via Galileo Galilei, 00044 Frascati, Italy

5 Agenzia Spaziale Italiana, Viale Liegi 26, 00198 Roma, Italy

6 Astrophysics Group, Cavendish Laboratory, University of Cambridge, J J Thomson Avenue, Cambridge CB3 OHE, UK

7 Astrophysics \& Cosmology Research Unit, School of Mathematics, Statistics \& Computer Science, University of KwaZulu-Natal, Westville Campus, Private Bag X54001, 4000 Durban, South Africa

8 CITA, University of Toronto, 60 St. George St., Toronto, ON M5S 3H8, Canada

9 CNRS, IRAP, 9 Av. colonel Roche, BP 44346, 31028 Toulouse Cedex 4, France

10 California Institute of Technology, Pasadena, California, USA

11 Centre for Theoretical Cosmology, DAMTP, University of Cambridge, Wilberforce Road, Cambridge CB3 0WA UK

12 Centro de Estudios de Física del Cosmos de Aragón (CEFCA), Plaza San Juan 1, planta 2, 44001 Teruel, Spain

13 Computational Cosmology Center, Lawrence Berkeley National Laboratory, Berkeley, California, USA

14 Consejo Superior de Investigaciones Científicas (CSIC), Madrid, Spain

15 DSM/Irfu/SPP, CEA-Saclay, 91191 Gif-sur-Yvette Cedex, France

16 DTU Space, National Space Institute, Technical University of Denmark, Elektrovej 327, 2800 Kgs. Lyngby, Denmark

17 Département de Physique Théorique, Université de Genève, 24 Quai E. Ansermet, 1211 Genève 4, Switzerland

18 Departamento de Física Fundamental, Facultad de Ciencias, Universidad de Salamanca, 37008 Salamanca, Spain
19 Departamento de Física, Universidad de Oviedo, Avda. Calvo Sotelo s/n, 33007 Oviedo, Spain

20 Department of Astronomy and Astrophysics, University of Toronto, 50 Saint George Street, Toronto, Ontario, Canada

21 Department of Astrophysics/IMAPP, Radboud University Nijmegen, PO Box 9010, 6500 GL Nijmegen, The Netherlands

22 Department of Electrical Engineering and Computer Sciences, University of California, Berkeley, California, USA

23 Department of Physics \& Astronomy, University of British Columbia, 6224 Agricultural Road, Vancouver, British Columbia, Canada

24 Department of Physics and Astronomy, Dana and David Dornsife College of Letter, Arts and Sciences, University of Southern California, Los Angeles, CA 90089, USA

25 Department of Physics and Astronomy, University College London, London WC1E 6BT, UK

26 Department of Physics, Gustaf Hällströmin katu 2a, University of Helsinki, Helsinki, Finland

27 Department of Physics, Princeton University, Princeton, New Jersey, USA

28 Department of Physics, University of California, One Shields Avenue, Davis, California, USA

29 Department of Physics, University of California, Santa Barbara, California, USA

30 Department of Physics, University of Illinois at Urbana-Champaign, 1110 West Green Street, Urbana, Illinois, USA

31 Dipartimento di Fisica e Astronomia G. Galilei, Università degli Studi di Padova, via Marzolo 8, 35131 Padova, Italy

32 Dipartimento di Fisica e Scienze della Terra, Università di Ferrara, via Saragat 1, 44122 Ferrara, Italy

33 Dipartimento di Fisica, Università La Sapienza, P.le A. Moro 2, 00185 Roma, Italy

34 Dipartimento di Fisica, Università degli Studi di Milano, via Celoria, 16, 20133 Milano, Italy

35 Dipartimento di Fisica, Università degli Studi di Trieste, via A. Valerio 2, 34127 Trieste, Italy

36 Dipartimento di Fisica, Università di Roma Tor Vergata, via della Ricerca Scientifica 1, 00133 Roma, Italy

37 Discovery Center, Niels Bohr Institute, Blegdamsvej 17, 2100 Copenhagen, Denmark

38 Dpto. Astrofísica, Universidad de La Laguna (ULL), 38206 La Laguna, Tenerife, Spain

39 European Space Agency, ESAC, Planck Science Office, Camino bajo del Castillo s/n, Urbanización Villafranca del Castillo, 28691 Villanueva de la Cañada, Madrid, Spain

40 European Space Agency, ESTEC, Keplerlaan 1, 2201 AZ Noordwijk, The Netherlands

41 Helsinki Institute of Physics, Gustaf Hällströmin katu 2, University of Helsinki, 00014 Helsinki, Finland

42 INAF - Osservatorio Astronomico di Padova, Vicolo dell'Osservatorio 5, 35122 Padova, Italy

43 INAF - Osservatorio Astronomico di Roma, via di Frascati 33, 00040 Monte Porzio Catone, Italy

44 INAF - Osservatorio Astronomico di Trieste, via G.B. Tiepolo 11, 34131 Trieste, Italy

45 INAF/IASF Bologna, via Gobetti 101, 40129 Bologna, Italy

46 INAF/IASF Milano, via E. Bassini 15, 20133 Milano, Italy

47 INFN, Sezione di Bologna, via Irnerio 46, 40126 Bologna, Italy

48 INFN, Sezione di Roma 1, Università di Roma Sapienza, Piazzale Aldo Moro 2, 00185 Roma, Italy

49 IPAG: Institut de Planétologie et d'Astrophysique de Grenoble, Université Joseph Fourier, Grenoble 1/CNRS-INSU, UMR 5274, 38041 Grenoble, France

50 IUCAA, Post Bag 4, Ganeshkhind, Pune University Campus, 411 007 Pune, India

51 Imperial College London, Astrophysics group, Blackett Laboratory, Prince Consort Road, London, SW7 2AZ, UK

52 Infrared Processing and Analysis Center, California Institute of Technology, Pasadena, CA 91125, USA 
53 Institut Néel, CNRS, Université Joseph Fourier Grenoble I, 25 rue des Martyrs, 38042 Grenoble, France

54 Institut Universitaire de France, 103 Bd Saint-Michel, 75005 Paris, France

55 Institut d'Astrophysique Spatiale, CNRS (UMR8617) Université Paris-Sud 11, Bâtiment 121, 91405 Orsay, France

56 Institut d'Astrophysique de Paris, CNRS (UMR7095), 98bis Bd Arago, 75014 Paris, France

57 Institute for Space Sciences, 077125 Bucharest-Magurale, Romania

58 Institute of Astronomy and Astrophysics, Academia Sinica, 10617 Taipei, Taiwan

59 Institute of Astronomy, University of Cambridge, Madingley Road, Cambridge CB3 0HA, UK

${ }^{60}$ Institute of Mathematics and Physics, Centre for Cosmology, Particle Physics and Phenomenology, 1348 Louvain University, Louvain-la-Neuve, Belgium

61 Institute of Theoretical Astrophysics, University of Oslo, Blindern, 0315 Oslo, Norway

${ }^{62}$ Instituto de Astrofísica de Canarias, C/Vía Láctea s/n, 38200 La Laguna, Tenerife, Spain

63 Instituto de Física de Cantabria (CSIC-Universidad de Cantabria), Avda. de los Castros s/n, 39005 Santander, Spain

64 Jet Propulsion Laboratory, California Institute of Technology, 4800 Oak Grove Drive, Pasadena, California, USA

65 Jodrell Bank Centre for Astrophysics, Alan Turing Building, School of Physics and Astronomy, The University of Manchester, Oxford Road, Manchester, M13 9PL, UK

${ }^{66}$ Kavli Institute for Cosmology Cambridge, Madingley Road, Cambridge, CB3 OHA, UK

${ }^{67}$ LAL, Université Paris-Sud, CNRS/IN2P3, 91898 Orsay, France

${ }^{68}$ LERMA, CNRS, Observatoire de Paris, 61 Avenue de l'Observatoire, 75014 Paris, France

69 Laboratoire AIM, IRFU/Service d'Astrophysique - CEA/DSM CNRS - Université Paris Diderot, Bât. 709, CEA-Saclay, 91191 Gifsur-Yvette Cedex, France

${ }^{70}$ Laboratoire Traitement et Communication de l'Information, CNRS (UMR 5141) and Télécom ParisTech, 46 rue Barrault, 75634 Paris Cedex 13, France

71 Laboratoire de Physique Subatomique et de Cosmologie, Université Joseph Fourier Grenoble I, CNRS/IN2P3, Institut National Polytechnique de Grenoble, 53 rue des Martyrs, 38026 Grenoble Cedex, France
72 Laboratoire de Physique Théorique, Université Paris-Sud 11 \& CNRS, Bâtiment 210, 91405 Orsay, France

73 Lawrence Berkeley National Laboratory, Berkeley, California, USA

74 Max-Planck-Institut für Astrophysik, Karl-Schwarzschild-Str. 1, 85741 Garching, Germany

75 Max-Planck-Institut für Extraterrestrische Physik, Giessenbachstraße, 85748 Garching, Germany

76 McGill Physics, Ernest Rutherford Physics Building, McGill University, 3600 rue University, Montréal, QC, H3A 2T8, Canada

77 MilliLab, VTT Technical Research Centre of Finland, Tietotie 3, 02044 Espoo, Finland

78 Niels Bohr Institute, Blegdamsvej 17, 2100 Copenhagen, Denmark

79 Observational Cosmology, Mail Stop 367-17, California Institute of Technology, Pasadena, CA, 91125, USA

${ }^{80}$ Optical Science Laboratory, University College London, Gower Street, London, UK

81 SB-ITP-LPPC, EPFL, 1015, Lausanne, Switzerland

82 SISSA, Astrophysics Sector, via Bonomea 265, 34136, Trieste, Italy

83 School of Physics and Astronomy, Cardiff University, Queens Buildings, The Parade, Cardiff, CF24 3AA, UK

${ }^{84}$ School of Physics and Astronomy, University of Nottingham, Nottingham NG7 2RD, UK

85 Space Sciences Laboratory, University of California, Berkeley, California, USA

86 Special Astrophysical Observatory, Russian Academy of Sciences, Nizhnij Arkhyz, Zelenchukskiy region, 369167 KarachaiCherkessian Republic, Russia

87 Stanford University, Dept of Physics, Varian Physics Bldg, 382 via Pueblo Mall, Stanford, California, USA

88 Sub-Department of Astrophysics, University of Oxford, Keble Road, Oxford OX1 3RH, UK

89 Theory Division, PH-TH, CERN, 1211 Geneva 23, Switzerland

90 UPMC Univ. Paris 06, UMR7095, 98bis Boulevard Arago, 75014 Paris, France

91 Université de Toulouse, UPS-OMP, IRAP, 31028 Toulouse Cedex 4, France

92 University of Granada, Departamento de Física Teórica y del Cosmos, Facultad de Ciencias, 1807 Granada, Spain

93 University of Miami, Knight Physics Building, 1320 Campo Sano Dr., Coral Gables, Florida, USA

94 Warsaw University Observatory, Aleje Ujazdowskie 4, 00-478 Warszawa, Poland 